NBER WORKING PAPER SERIES

\title{
A THEORY OF FIRM DECLINE
}

Gian Luca Clementi

Thomas F. Cooley

Sonia Di Giannatale

Working Paper 15192

http://www.nber.org/papers/w15192

\author{
NATIONAL BUREAU OF ECONOMIC RESEARCH \\ 1050 Massachusetts Avenue \\ Cambridge, MA 02138 \\ July 2009
}

We are grateful to Dave Backus, Heski Bar-Isaac, Alberto Bisin,Andre De Souza, Kose John, Boyan Jovanovic, Claudio Loderer, Bob Lucas, Narayana Kocherlakota and Tom Sargent, as well seminar attendants at the Minneapolis Fed, NYU, the 2008 Midwest Macro Conference in Philadelphia, the 2008 meetings of the Society for Economic Dynamics (Cambridge, MA) and European Economic Association (Milan), and the 2009 Econometric Society Meeting in Boston for their comments and suggestions. The views expressed herein are those of the author(s) and do not necessarily reflect the views of the National Bureau of Economic Research.

NBER working papers are circulated for discussion and comment purposes. They have not been peerreviewed or been subject to the review by the NBER Board of Directors that accompanies official NBER publications.

(C) 2009 by Gian Luca Clementi, Thomas F. Cooley, and Sonia Di Giannatale. All rights reserved. Short sections of text, not to exceed two paragraphs, may be quoted without explicit permission provided that full credit, including $\odot$ notice, is given to the source. 
A Theory of Firm Decline

Gian Luca Clementi, Thomas F. Cooley, and Sonia Di Giannatale

NBER Working Paper No. 15192

July 2009

JEL No. E0,L11

\begin{abstract}
$\underline{\text { ABSTRACT }}$
We study the problem of an investor who buys an equity stake in an entrepreneurial venture, under the assumption that the former cannot monitor the latter's operations. The dynamics implied by the optimal incentive scheme is rich and quite different from that induced by other models of repeated moral hazard. In particular, our framework generates a rationale for firm decline. As young firms accumulate capital, the claims of both investor (outside equity) and entrepreneur (inside equity) increase. At some juncture, however, even as the latter keeps on growing, invested capital and firm value start declining and so does the value of outside equity. The reason is that incentive provision is costlier the wealthier the entrepreneur (the greater is inside equity). In turn, this leads to a decline in the constrained-efficient level of effort and therefore to a drop in the return to investment.
\end{abstract}

Gian Luca Clementi

New York University

Stern School of Business

44 West Fourth Street

New York, NY 10012

gclement@stern.nyu.edu

Thomas F. Cooley

Department of Economics

Stern School of Business

44 West 4th Street, Room 7-88

New York, NY 10012-1126

and NBER

tcooley@ stern.nyu.edu
Sonia Di Giannatale

Centro de Investigacion y Docencia Economicas

Mexico D.F.

Mexico

sonia.digiannatale@cide.edu 


\section{Introduction}

Think of an investor who provides seed financing to an entrepreneurial firm. The success of her investment will depend crucially on the effort that the entrepreneur puts in. Yet, the incentives of the two individuals are not perfectly aligned, as the entrepreneur bears the whole cost of such effort while sharing the pecuniary returns with the investor. Since monitoring the entrepreneur's conduct is prohibitively expensive, the investor will find it optimal to implement an incentive scheme that links rewards to observables. We are interested in characterizing the implications of such scheme for the dynamics of firm size and value, as well as the latter's split between the two agents.

The problem just described can be conveniently cast as a model of repeated bilateral exchange with hidden action, along the lines of Spear and Srivastava (1987) and Wang (1997). The only caveat is that in their models there is no notion of important features of firm dynamics such as production and capital accumulation. The purpose of this paper is to explicitly model both of them. We do so by assuming that the entrepreneur is equipped with a production function that exhibits decreasing returns and is hit by multiplicative shocks whose probability distribution depends in a natural way on managerial effort.

The incentive scheme chosen by the investor belongs to the set of constrainedefficient allocations, which in turn consist of sequences of effort provision, payouts, and investment, that maximize the value of the investor's claim (outside equity) for given rewards to the entrepreneur.

The main insight generated by our analysis is a rationale for firm decline, which follows from the negative relation between the marginal value of investment and the value of the entrepreneur's claim (inside equity). Since the entrepreneur's utility is additively separable in consumption and effort and displays constant relative risk aversion to consumption bets, incentive provision is costlier, the greater the value of the entrepreneur's claim to the venture's cash flows. ${ }^{1}$ Everything else equal, this means that the higher the entrepreneur's wealth, the lower the constrained-efficient level of effort provision. By reducing the likelihood of a high productivity shock, this results in a lower marginal value of investment.

A lending contract assigns to the entrepreneur a level of capital and a claim to

\footnotetext{
${ }^{1}$ This is a well-known property, exploited by Spear and Wang (2005) and Wang (2006) to model the optimal termination of employment contracts and by Newman (2007) to study the relationship between wealth and occupational choice.
} 
future cash flows. For consistency with the empirical evidence on the relative size of entrant firms, assume that the initial capital is small (in a sense to be made precise later). Then, the typical firm dynamics predicted by our model is as follows. Given decreasing returns, early on the marginal product of capital is high. Since further infusions of capital from the investor are ruled out by assumption, firm size gradually increases over time, thanks to the investment of retained earnings. Optimal incentive provision dictates that on average inside equity increases as well. These forces have countervailing effects on the marginal value of managerial effort, positive for capital and negative for entrepreneurial wealth. Eventually, the latter dominates. Capital and effort start declining, and so does firm value.

When the elasticity of intertemporal substitution is greater than one and agents discount future utility at the same rate, in the limit all cash flows accrue to the entrepreneur. Firm size, effort, and inside equity converge to a constant. When the entrepreneur is either relatively more impatient or her EIS coefficient is smaller than 1, the model allows for a non-degenerate stationary distribution of firm size, firm value, and its split between the two agents.

We find that the qualitative features of firm dynamics we have just described survive the generalization to the scenario in which productivity shocks are persistent.

This paper contributes to a small but growing literature that explores the implications of moral hazard for firm dynamics. Albuquerque and Hopenhayn (2004) and Cooley, Marimon, and Quadrini (2004) consider scenarios where the entrepreneur has limited commitment, while Clementi and Hopenhayn (2006), Brusco and Ropero (2007), Quadrini (2003) and DeMarzo, Fishman, He, and Wang (2008) study the case of hidden information. All of these models, as well as other theories of firm dynamics such as Jovanovic (1982) and Hopenhayn (1992), are able to rationalize the negative correlation between age and the exit hazard rate that was documented for relatively young firms. None of them, however, is consistent with the evidence, provided by Aggarwal and Gort (1996, 2002), that for older firms the exit hazard rate increases with age, irrespective of the industry life-cycle phase.

This caveat does not apply to our theory. For every cohort of firms whose dynamics are described by our contract, there is a point in time after which average firm size and value decline with age. Assuming a a constant outside value for firms' assets, this would result in a positive association between age and exit hazard rate.

Our paper also belongs to a large literature, started by Holmstrom (1979), that analyzes constrained-efficient allocations in principal-agent models with hidden action. 
Our work is part of the more recent tradition which began with Rogerson (1985b), that explicitly considers repeated relationships. A number of papers in this line of work, among which Wang (1997) and Clementi, Cooley, and Wang (2006), have interpreted the principal-agent relationship as one between shareholders and executives. This alternative interpretation is also valid for our model.

Finally, our framework also has close ties to equilibrium models that allow for capital accumulation in environments where market incompleteness is caused by moral hazard. Among these, the closest work is by Bohacek (2005), who provides conditions under which an economy à la Atkeson and Lucas (1995) admits a stationary and ergodic distribution of consumption. Other papers in this class include Marcet and Marimon (1992), Khan and Ravikumar (2001), and Espino (2005).

The remainder of the paper is organized as follows. The model is introduced in Section 2. In Section 3 we characterize the constrained-efficient allocations that arise in two special cases of our environment, namely one with no dynamics and one with dynamics but no capital accumulation. That analysis helps building intuition for the results illustrated in Section 4, where the general model is considered. In Section 5 we discuss the empirical relevance of our theory. Section 6 is dedicated to comparative statics exercises. Section 7 considers the scenario in which shocks are autocorrelated. Finally, Section 8 concludes.

\section{Model}

Time is discrete and is indexed by $t=1,2, \ldots$ There are two agents, who we will refer to as investor and entrepreneur, respectively. The latter is endowed with a production technology, that produces a homogeneous good with capital as the only input. Output $\left(y_{t}\right)$ is given by

$$
y_{t}=\theta_{t} f\left(k_{t}\right),
$$

where $k_{t} \in[\underline{k}, \bar{k}] \in \Re_{+}$and $\theta_{t} \in \Theta \subseteq \Re_{+}$is a random variable distributed according to the time-invariant distribution function $G\left(\theta_{t} \mid a_{t}\right)$. The variable $a_{t} \in A \equiv[\underline{a}, \bar{a}] \in \Re_{+}$ denotes managerial effort. We assume that $G$ has a density denoted by $g$, which is twice continuously differentiable with respect to $a$, and that $\Theta$ is compact.

While the output of the production process is public knowledge, the effort exerted by the entrepreneur is her private information.

At the outset, the investor provides the entrepreneur with capital $k_{1}$. We do not model the bargaining process by which the two agents agree on such level of capital 
and on a particular split of the surplus. We also assume that any further investment must be financed with resources produced internally. It follows that, at all $t$,

$$
c_{t}+x_{t} \leq \theta_{t} f\left(k_{t}\right)
$$

where $c_{t} \geq 0$ is the entrepreneur's consumption and $x_{t}$ denotes investment. Since condition (1) requires the payoffs to the investor to be non-negative, we will refer to it as the limited liability constraint. The law of motion for capital is the usual one:

$$
k_{t+1}=(1-\delta) k_{t}+x_{t}
$$

where $\delta \in(0,1)$ denotes the depreciation rate. The last two conditions imply the following resource constraint:

$$
c_{t} \leq \theta_{t} f\left(k_{t}\right)+(1-\delta) k_{t}-k_{t+1}
$$

We assume that the investor is risk-neutral, while the entrepreneur is risk-averse. The latter's static preferences are represented by the utility function $u\left(c_{t}\right)-a$. We posit that $u(\cdot)$ belongs to the CRRA class, i.e. $u(c)=\frac{c^{1-\chi}}{1-\chi}, \chi>0, \chi \neq 1$. Agents discount future utility streams at the common rate $\frac{1}{\beta}-1$, where $\beta \in(0,1){ }^{2}$

We allow for employ history-dependent pure strategies. If we let $h^{0}$ denote the empty history, then the history at time $t \geq 1$ is given by the sequence $h^{t}=$ $h^{0} \cup\left\{\left(\theta_{s}, k_{s}\right)\right\}_{s=1}^{t}$. The investor's task is to offer the entrepreneur an incentive scheme (contract) $\sigma=\left\{a_{t}\left(h^{t-1}, k_{t}\right), c_{t}\left(h^{t}\right), k_{t+1}\left(h^{t-1}, k_{t}\right)\right\}_{t=1}^{\infty}$. This notation reflects the assumption, typical in neoclassical macroeconomics, that investment is chosen at the beginning of every period, before the realization of the shock. ${ }^{3}$ The timing is summarized in Figure 1.

Given $h^{t}$ and $k_{t+1}$, the continuation profile of a contract $\sigma$ from date $t+1$ on is denoted as $\sigma \mid h^{t}, k_{t+1}$. Conditional on the entrepreneur following the actions recommended by such profile, her continuation value and the investor's are denoted by $\omega\left(\sigma \mid h^{t}, k_{t+1}\right)$ and $v\left(\sigma \mid h^{t}, k_{t+1}\right)$, respectively.

A contract $\sigma$ is said to be feasible if, at all times and after any history, effort recommendations belong to the set $A$ and the resource constraint is satisfied. More formally,

\footnotetext{
${ }^{2}$ In Section 6 we will relax this restriction by considering the case in which the entrepreneur is relatively more impatient.

${ }^{3}$ In Section 3 we comment briefly on how the constrained-efficient allocation would change, if we assumed that investment was chosen after the realization of the shock.
} 


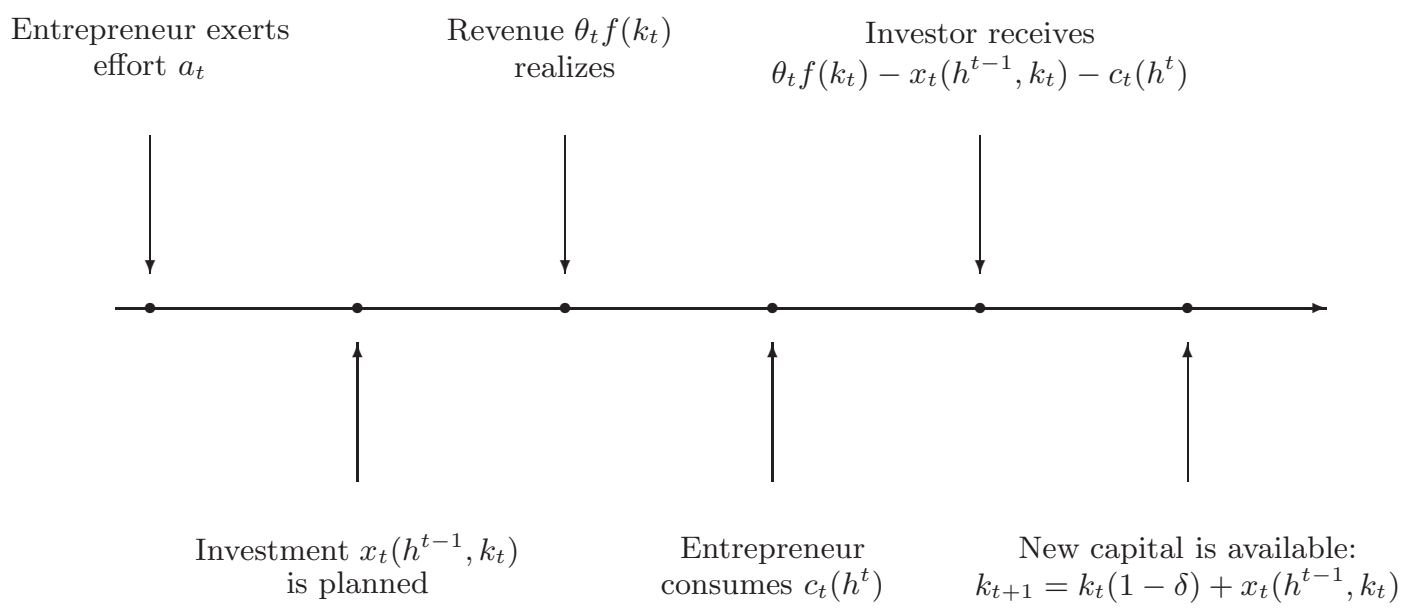

Figure 1: Timing in period $t$.

Definition 1 A contract $\sigma$ is feasible if, for all $t \geq 1$,

$$
a_{t}\left(h^{t-1}, k_{t}\right) \in A, \forall h^{t-1}, k_{t},
$$

and

$$
0 \leq c_{t}\left(h^{t}\right) \leq \theta_{t} f\left(k_{t}\right)+(1-\delta) k_{t}-k_{t+1}\left(h^{t-1}, k_{t}\right), \forall h^{t}
$$

The temporary incentive compatibility constraint rules out one-shot deviations at all dates and after all histories and can be formally stated as follows:

Definition 2 A contract $\sigma$ is temporary incentive compatible if, $\forall t \geq 1$ and $\forall h^{t-1}, k_{t}$,

$$
a_{t}\left(h^{t-1}, k_{t}\right) \in \arg \max _{a} \int_{\theta}\left\{u\left(c_{t}\left(h^{t}\right)\right)-a+\beta \omega\left(\sigma \mid h^{t}, k_{t+1}\right)\right\} g\left(\theta_{t} \mid a\right) d \theta_{t} .
$$

Given our assumptions, the unimprovability principle guarantees that condition (4) also rules out any arbitrary sequence of deviations from the investor's effort recommendation plan. ${ }^{4}$

The fact that the set $A$ is a connected subset of $\Re_{+}$suggests that rewriting (4) as a first-order condition may be handy. In the literature, this is known as the first-order approach, which is not universally valid. To ensure its validity, we follow Rogerson (1985a) and Spear and Srivastava (1987) in assuming that the Monotone Likelihood Ratio Property and the Convexity of the Conditional Distribution Condition hold.

\footnotetext{
${ }^{4}$ See Green (1987).
} 
Let $\Omega$ be the set of pairs $(k, \omega)$ such that there exists a feasible and incentive compatible contract that delivers $\omega$, given $k$. That is, for $\Delta \in \Re^{2}$ non-empty and compact, let

$$
\Omega \equiv\left\{(k, \omega) \in \Delta \mid \exists \sigma \text { s.t. (2), (3), (4), } k_{1}=k \text {, and } \omega\left(\sigma \mid h^{0}\right)=\omega\right\}
$$

For every $(k, \omega) \in \Omega$, define $\Phi(k, \omega)$ as the set of the investor's expected discounted utilities that can be generated by feasible and incentive compatible contracts delivering $\omega$ to the entrepreneur for given $k$. That is,

$$
\Phi(k, \omega)=\left\{v\left(\sigma \mid h^{0}\right) \mid \exists \sigma \text { s.t. (2), (3), (4), } k_{1}=k, \text { and } \omega\left(\sigma \mid h^{0}\right)=\omega\right\} .
$$

Proposition $1 \Phi(k, w)$ is compact $\forall(k, \omega) \in \Omega .^{5}$

For given $(k, \omega)$, the investor's problem is to choose a feasible and incentive compatible contract $\sigma$ that attains the maximum element in $\Phi(k, \omega)$. Denote such element as $v^{*}(k, \omega)$.

Proposition 2 shows that $v^{*}(k, \omega)$ is a fixed point of the operator $T$, which maps the space of bounded and continuous functions $v: \Omega \rightarrow \Re$ into itself, with the sup norm, and is given by

$$
\begin{aligned}
T(v)(k, \omega) \equiv & \max _{a^{*}, k^{\prime}, c(\theta), \omega^{\prime}(\theta)} \int_{\Theta}\left\{\theta f(k)-c(\theta)-k^{\prime}+(1-\delta) k+\beta v\left(k^{\prime}, \omega^{\prime}(\theta)\right)\right\} g\left(\theta \mid a^{*}\right) d \theta \\
\text { s.t. } & \int_{\Theta}\left\{u(c(\theta))-a+\beta \omega^{\prime}(\theta)\right\} g\left(\theta \mid a^{*}\right) d \theta=\omega, \\
& a^{*} \in \arg \max _{a \in A} \int_{\Theta}\left\{u(c(\theta))-a+\beta \omega^{\prime}(\theta)\right\} g(\theta \mid a) d \theta, \\
& 0 \leq c(\theta) \leq \theta f(k)-k^{\prime}+k(1-\delta) \quad \forall \theta \in \Theta, \\
& \left(k^{\prime}, \omega^{\prime}(\theta)\right) \in \Omega \forall \theta \in \Theta .
\end{aligned}
$$

Proposition $2 v^{*}(k, \omega)=T\left(v^{*}\right)(k, \omega)$ for all $(k, \omega) \in \Omega$.

Since $T$ satisfies Blackwell's sufficient conditions for a contraction, the contraction mapping theorem ensures that the fixed point is unique. Solving for it also yields policy functions for recommended effort $a(k, \omega)$, entrepreneur's cash flows $c(k, \omega, \theta)$, and continuation utility $\omega^{\prime}(k, \omega, \theta)$, which can be used to recover the constrainedefficient contract in a straightforward manner.

\footnotetext{
${ }^{5}$ All the results and the proofs not included in the main text can be found in Appendix A.
} 
By adapting results from Abreu, Pierce, and Stacchetti (1990) to our environment, Proposition 3 shows that $\Omega$ is the fixed point of the set operator $B$, defined as follows.

Definition 3 For any arbitrary $\Sigma \in \Re^{2}$,

$$
B(\Sigma)=\left\{(k, w) \mid \exists\left\{a, k^{\prime}, c(\theta), \omega^{\prime}(\theta)\right\} \text { s.t. (5), (6), (7), and }\left(k^{\prime}, \omega^{\prime}(\theta)\right) \in \Sigma, \forall \theta\right\} .
$$

Proposition 3 (a) $\Omega=B(\Omega)$. (b) Take any closed and bounded set $X_{0}$ such that $X_{0} \subseteq \Omega_{0}$. Let $X_{n+1}=B\left(X_{n}\right)$, for $n=0,1,2, \ldots$ Then, $\lim _{n \rightarrow \infty} X_{n}=\Omega$.

Proposition 3 also shows that the sequence constructed by iterating on $B$ starting with $X_{0} \subseteq \Omega_{0}$ converges to the set $\Omega$. Since an analytical characterization of the constrained Pareto-optimal contract is not possible, this result will be useful in the numerical approximation of the allocation that results from it.

In the next section, we make assumptions about functional forms and parameters that we will maintain for the remainder of the paper. The algorithms that were designed to approximate the set $\Omega$ and the function $v(k, \omega)$ are described in Appendix B.

\subsection{Numerical Implementation}

We assume that the production function is $f(k)=k^{\alpha}, \alpha \in(0,1)$. Furthermore, we posit that $A=[0, \bar{a}]$ and $\Theta=\left\{\theta_{l}, \theta_{h}\right\}$, with $\theta_{h}>\theta_{l}$ and $G\left(\theta_{l} \mid a\right)=e^{-a}$. ${ }^{6}$ While this choice of conditional distribution is mostly dictated by tractability, it has appealing features. The probability of a good outcome is zero if no effort is exerted, and goes to 1 as effort grows unboundedly large. Furthermore, the marginal effect of effort on the probability of success is decreasing in the effort itself. From now on, all variables that are contingent on the shock realization will be denoted with the subscripts $l$ or $h$.

Under the assumptions listed above, the Bellman equation is

$$
\begin{gathered}
v(k, \omega)=\max _{a^{*}, k^{\prime},\left\{c_{i}, \omega_{i}\right\}_{i=h, l}}\left(1-e^{-a^{*}}\right)\left[\theta_{h} k^{\alpha}-c_{h}+\beta v\left(k^{\prime}, \omega_{h}\right)\right]+e^{-a^{*}}\left[\theta_{l} k^{\alpha}-c_{l}+\beta v\left(k^{\prime}, \omega_{l}\right)\right] \\
+k(1-\delta)-k^{\prime}
\end{gathered}
$$

\footnotetext{
${ }^{6}$ The upper bound $\bar{a}$ will be chosen so as to ensure it never binds.
} 


$$
\begin{array}{ll}
\text { s.t. } & \left(1-e^{-a^{*}}\right)\left[u\left(c_{h}\right)+\beta \omega_{h}\right]+e^{-a^{*}}\left[u\left(c_{l}\right)+\beta \omega_{l}\right]-a^{*}=\omega, \\
& a^{*} \in \arg \max _{a \in A}\left(1-e^{-a}\right)\left[u\left(c_{h}\right)+\beta \omega_{h}\right]+e^{-a}\left[u\left(c_{l}\right)+\beta \omega_{l}\right]-a, \\
& 0 \leq c_{i} \leq \theta_{i} k^{\alpha}-k^{\prime}+(1-\delta) k \forall i=h, l, \\
& \left(k^{\prime}, \omega_{i}\right) \in \Omega \forall i=h, l .
\end{array}
$$

For all $(k, \omega) \in \Omega, v(k, \omega)$ yields the expected discounted value of the cash flows that will accrue to the investor when the current capital stock is $k$ and promised utility is $\omega$. For this reason, it can be thought of as outside equity. We denote as $C(k, \omega)$ the expected discounted cost to the investor of delivering $\omega$ to the entrepreneur, when the current capital stock is $k$. The function $C(k, \omega)$ solves the following functional equation:

$$
C(k, \omega)=\left(1-e^{-a^{*}}\right)\left[c_{h}^{*}+\beta C\left(k^{\prime *}, \omega_{h}^{*}\right)\right]+e^{-a^{*}}\left[c_{l}^{*}+\beta C\left(k^{*}, \omega_{l}^{*}\right)\right],
$$

where the asterisks designate the optimal choices generated by problem (P1). Since $C(k, \omega)$ is also the expected discounted value of the cash flows that will accrue to the entrepreneur, we will often refer to it as the value of inside equity.

Unless stated otherwise, the parameter values used to compute the examples in the remainder of the paper are those reported in Table 1. Even though we set $\beta, \chi, \alpha$, and $\delta$ to values that are standard in the macroeconomics literature, we wish to emphasize that by no means should this be considered a calibration exercise.

The analysis that follows will show that the choice of $\chi$ is particularly relevant. In our benchmark, we set $\chi=0.5$ because of the rapidly amassing experimental evidence in favor this value. ${ }^{7}$ Section 4.2 discusses how the constrained-efficient allocation changes when $\chi>1$.

\begin{tabular}{ccccccccc}
\hline$\underline{k}$ & $\bar{k}$ & $\beta$ & $\chi$ & $\alpha$ & $\delta$ & $\theta_{h}$ & $\theta_{l}$ & $\underline{\omega}$ \\
0 & 3.5 & 0.95 & 0.5 & 0.3 & 0.1 & 1.5 & 0.4 & 10.5 \\
\hline
\end{tabular}

Table 1: Parameter Values.

\section{The Optimal Contract Without Capital Accumulation}

The purpose of this section is to illustrate the properties of the optimal incentive schemes that obtain in two scenarios that are special cases of the environment de-

\footnotetext{
${ }^{7}$ See for example Choi, Fisman, Gale, and Kariv (2007) and references therein cited.
} 
scribed above. The intuition gained here will be helpful in Section 4, where we will tackle the general case.

We start by considering the scenario where the relationship between investor and entrepreneur lasts only one period. In Section 3.2 we will study the case of infinitely repeated interaction without capital accumulation.

\subsection{The Static Case}

The inverse of the utility function yields the cost to the investor of delivering a certain level of utility. Denote it as $c(u)$. Obviously, $c(u)$ is strictly increasing and strictly convex. For $\chi \neq 1$, we have that $c(u)=[(1-\chi) u]^{1 /(1-\chi)}$. For $\chi=1, c(u)=e^{u} .8$

We find it convenient to reformulate the problem to let the investor choose utilities rather than consumption allocations. With some abuse of notation, let $u_{i} \equiv u\left(c_{i}\right)$ define the utility the entrepreneur derives from consuming $c_{i}$, for $i=h, l$. For notational simplicity, let also $\bar{u}_{i}=u\left(\theta_{i} k^{\alpha}-\delta k\right)$. Finally, denote as $\bar{\omega}(k)$ and $\underline{\omega}(k)$ the supremum and infimum elements of the set of utilities that can be awarded to the entrepreneur by a feasible and incentive compatible contract, when the installed capital is $k$. The two points are characterized by Lemma 3 .

When capital in place is $k$, the value to the investor of delivering to the entrepreneur a utility $\omega, \underline{\omega}(k) \leq \omega \leq \bar{\omega}(k)$, is

$$
\begin{aligned}
\max _{u_{h}, u_{l}}\left(1-e^{-a^{*}}\right)\left[\theta_{h} k^{\alpha}-c\left(u_{h}\right)\right]+e^{-a^{*}}\left[\theta_{l} k^{\alpha}-c\left(u_{l}\right)\right]-\delta k, \\
\text { s.t. } \quad\left(1-e^{-a^{*}}\right) u_{h}+e^{-a^{*}} u_{l}-a^{*}=\omega, \\
a^{*}=\arg \max _{a \in A}\left(1-e^{-a}\right) u_{h}+e^{-a} u_{l}-a, \\
\underline{u} \leq u_{i} \leq u\left(\theta_{i} k^{\alpha}-\delta k\right), \quad \forall i=h, l,
\end{aligned}
$$

where $\underline{u}=0$ if $\chi<1$ and $\underline{u}=-\infty$ otherwise. The constrained-efficient allocations are characterized formally in Proposition 5. Here we describe their main features with the help of Figure 2.

From the incentive compatibility constraint it follows immediately that the recommended effort is $a^{*}=\log \left(u_{h}-u_{l}\right)$. A higher effort is implementable only by increasing $s \equiv u_{h}-u_{l}$, the gap between contingent rewards.

Combining (13) with the expression for the effort recommendation, the optimiza-

\footnotetext{
${ }^{8}$ Notice that for $\chi<1, c: \Re^{+} \rightarrow \Re^{+}$. For $\chi=1, c: \Re \rightarrow \Re^{+}$. For $\chi>1 c: \Re^{-} \rightarrow \Re^{+}$.
} 

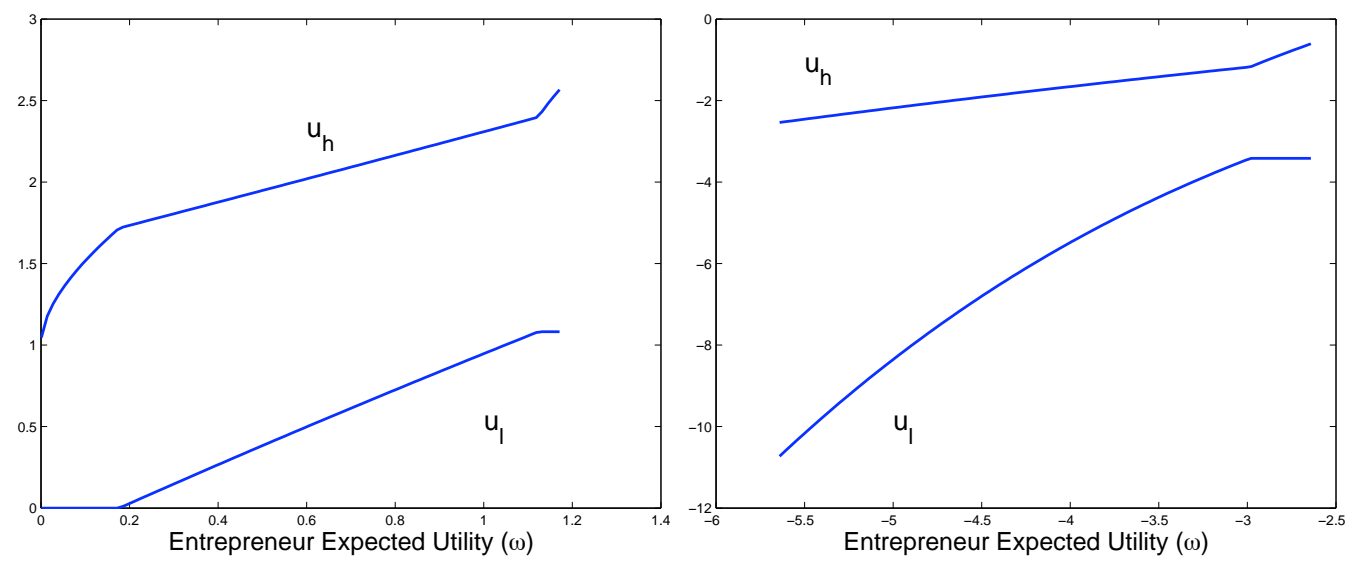

Figure 2: Static Model. Policies for $\chi=1 / 2$ (left) and $\chi=2$ (right).

tion problem reduces to

$$
\begin{aligned}
\max _{s \geq 1}\left(1-\frac{1}{s}\right) & {\left[\theta_{h} k^{\alpha}-c\left(u_{h}\right)\right]+\frac{1}{s}\left[\theta_{l} k^{\alpha}-c\left(u_{l}\right)\right]-\delta k, } \\
\text { s.t. } & u_{h}=1+\omega+\log (s), \\
& u_{l}=1+\omega+\log (s)-s, \\
& \underline{u} \leq u_{i} \leq u\left(\theta_{i} k^{\alpha}-\delta k\right) \forall i=h, l .
\end{aligned}
$$

Necessary and sufficient condition for an interior solution is

$$
\frac{1}{s^{2}}\left[\theta_{h}-\theta_{l}\right] k^{\alpha}=\frac{1}{s^{2}}\left[c\left(u_{h}\right)-c\left(u_{l}\right)\right]+\frac{s-1}{s^{2}}\left[c^{\prime}\left(u_{h}\right)-c^{\prime}\left(u_{l}\right)\right] .
$$

Notice that the term $1 / s^{2}$ is the marginal increase in the probability of success induced by an increase in $s \equiv u_{h}-u_{l}$. The left-hand side of (14) is the expected marginal revenue gain resulting from the increase in $s$. The right-hand side is the marginal increase in the cost of compensating the entrepreneur. The first term reflects the increased probability of awarding $u_{h}$ rather than $u_{l}$. The second term reflects the marginal impact on the expected cost arising from an increase in the risk imposed on the entrepreneur. By strict concavity of the utility function, this term is positive as well.

As long as the solution is interior (i.e. $\underline{u}<u_{l}<\bar{u}$ ), Figure 2 shows that the recommended level of effort decreases with $\omega$. As promised utility increases, incentive provision becomes costlier. In turn, this implies that it is constrained-efficient to require the entrepreneur to exert a lower effort. Proposition 5 establishes that $\chi \geq 1 / 2$ is a sufficient condition (although not necessary) for this property to hold. 
Figure 2 also shows that $u_{l}$ is constant for relatively high $\omega$, signaling that the limited liability constraint binds in the low state. Proposition 5 shows that when $\bar{u}_{h}-\bar{u}_{l}>1$, as it is the case in our example, effort grows with $\omega$ over this range. When $\bar{u}_{h}-\bar{u}_{l} \leq 1$, effort is identically zero in the same region.

Finally, notice that, since utility is bounded below for $\chi<1$, efficient effort provision must be increasing with $\omega$ for low expected utility. In other words, since the constraint $u_{l} \geq 0$ binds in this region, larger values of $\omega$ can only be implemented by increasing $u_{h}$ and therefore the effort recommendation.

\subsection{The Dynamic Case}

We now consider the case in which the time horizon is infinite, but there is no capital accumulation. This scenario is very close to those analyzed by Spear and Srivastava (1987) and Wang (1997). It differs from the former in that we impose limited liability, i.e. the entrepreneur's cash-flow must be non-negative. It differs from the latter, since Wang assumes that the effort choice is binary and $u(c)$ belongs to the CARA class.

With slight abuse of notation, let $v(\omega)$ denote the value accruing to the investor when delivering utility $\omega$ to the entrepreneur and let $s \equiv u_{h}+\beta \omega_{h}-\left(u_{l}+\beta \omega_{l}\right)$.

We begin by considering the scenario for $\chi<1$, without the limited liability constraint. Without an upper bound to entrepreneur's consumption, all levels of

promised utility $\omega \geq 0$ could be implemented by a feasible and incentive compatible contract. It would suffice to require zero effort and award consumption $c[\omega /(1-\beta)]$ at all future dates. Therefore, we impose an arbitrary upper bound $\bar{\omega}>0$.

From the incentive compatibility constraint, it follows that recommended effort is $a^{*}=\log (s)$, for $s \geq 1$. Then, for all $\omega$ such that $0 \leq \omega \leq \bar{\omega}$, the value of outside equity $v(\omega)$ is the fixed point of the following operator:

$$
\begin{aligned}
& v(\omega)=\max _{s \geq 1, \omega_{h}, \omega_{l}}\left(1-\frac{1}{s}\right)\left[\theta_{h} k^{\alpha}-c\left(u_{h}\right)+\beta v\left(\omega_{h}\right)\right]+\left(\frac{1}{s}\right)\left[\theta_{l} k^{\alpha}-c\left(u_{l}\right)+\beta v\left(\omega_{l}\right)\right]-\delta k, \\
& \text { s.t. } u_{h}=\omega+1+\log (s)-\beta \omega_{h}, \\
& u_{l}=\omega+1+\log (s)-s-\beta \omega_{l}, \\
& u_{i} \geq 0 \forall i=h, l, \\
& 0 \leq \omega_{i} \leq \bar{\omega} \forall i=h, l .
\end{aligned}
$$

Proposition 4 Assume there is no limited liability constraint and $\chi<1$. Then, the value function $v(\omega)$ is strictly concave. Furthermore, for all $\omega$ such that the optimal 
choices $\omega_{l}$ and $\omega_{h}$ are interior:

(a) $\omega_{l}<\omega<\omega_{h}$;

(b) payments to the entrepreneur follow a sub-martingale.

Along with (15)-(16), the following conditions are necessary and sufficient for an interior solution to Problem (P2):

$$
c^{\prime}\left(u_{i}\right)=-v^{\prime}\left(\omega_{i}\right), \quad i=h, l
$$

and

$$
\left[\theta_{h}-\theta_{l}\right] k^{\alpha}+\beta\left[v\left(\omega_{h}\right)-v\left(\omega_{l}\right)\right]=\left[c\left(u_{h}\right)-c\left(u_{l}\right)\right]+(s-1)\left[c^{\prime}\left(u_{h}\right)-c^{\prime}\left(u_{l}\right)\right] .
$$

Condition (17) requires that the investor distributes contingent utility awards efficiently over time. Equation (18) is the analogue of condition (14). It indicates that the forces that shape the optimal spread of utilities across states are essentially the same as in the static case. The only difference is that the marginal benefit of increasing effort also depends on the difference between the investor's contingent continuation values.

When the solution is interior, the envelope condition is

$$
v^{\prime}(\omega)=-\left(1-\frac{1}{s_{i}}\right) c^{\prime}\left(u_{h}\right)-\left(\frac{1}{s_{i}}\right) c^{\prime}\left(u_{l}\right) .
$$

The latter, along with (17), implies that

$$
v^{\prime}(\omega)=\left(1-\frac{1}{s}\right) v^{\prime}\left(\omega_{h}\right)+\left(\frac{1}{s}\right) v^{\prime}\left(\omega_{l}\right)
$$

In turn, strict concavity of the value function implies that $\omega_{l}<\omega<\omega_{h}$.

Combining (19) and (17) yields a further condition, which is common to many repeated hidden action models and was first illustrated by Rogerson (1985b). Letting $s_{i}, u_{i h}$, and $u_{i l}$ denote next period's choices contingent on the current state of nature being $i$, we have that

$$
c^{\prime}\left(u_{i}\right)=\left(1-\frac{1}{s_{i}}\right) c^{\prime}\left(u_{i h}\right)+\left(\frac{1}{s_{i}}\right) c^{\prime}\left(u_{i l}\right), \quad i=h, l .
$$

By proposition 3 of Rogerson (1985b), claim (b) in Proposition 4 follows immediately from $(21)$.

Effort is not monotone in $\omega$. Here is why. It is obvious that $\omega=0$ is an absorbing state. For that level of promised utility, $a^{*}=0$ and $u_{l}=u_{h}=0$ at all times. For low 
but strictly positive values of $\omega$, the probability of ending up in such state is relatively high. In order to lower such probability, an increase in recommended effort should be implemented by lowering current utility at the benefit of the future. Unfortunately such strategy is compromised by the lower bound on utility. For $u_{l}=0$, the promisekeeping constraint reads $\omega=u_{h}+\beta \omega_{h}-1-\log \left[u_{h}+\beta\left(\omega_{h}-\omega_{l}\right)\right]$. Lowering $\omega_{l}$ towards its lower bound increases the probability of success and allows for higher utility in the high state. However, these gains are balanced by the fact that a bad shock will bring promised utility closer to 0 . As $\omega$ grows, the ability to provide incentives improves. For $\omega$ large enough, the effort choice becomes unconstrained. When this is the case, all of our numerical examples indicate that effort decreases with promised utility.

We now turn to the effects of introducing the limited liability constraint. Lemma 1 establishes that limited liability implies an upper bound for promised utility and that such upper bound is an absorbing state of the dynamical system.

Lemma 1 For $\bar{u}_{h}-\bar{u}_{l}>1$, the maximal element of the set $\Omega$ is $\bar{\omega}=\frac{1}{1-\beta}\left[\bar{u}_{h}-1-\right.$ $\left.\log \left(\bar{u}_{h}-\bar{u}_{l}\right)\right]$. The constrained-efficient contract is such that $u_{i}(\bar{\omega})=\bar{u}_{i}$ and $\omega_{i}(\bar{\omega})=\bar{\omega}$ for $i=h, l$. For $\bar{u}_{h}-\bar{u}_{l} \leq 1, \bar{\omega}=\frac{\bar{u}_{l}}{1-\beta}$, with $a^{*}(\bar{\omega})=0, u_{l}(\bar{\omega})=\bar{u}_{l}$ and $\omega_{l}(\bar{\omega})=\bar{\omega}$. In either case, $v(\bar{\omega})=0$.

Proof. The maximal element $\bar{\omega}$ is the fixed point of the APS operator $B(\omega)$ :

$$
\begin{gathered}
B(\omega) \equiv \max _{a \in A,\left\{u_{i}, \omega_{i}\right\}_{i=h, l}}\left(1-e^{-a}\right)\left[u_{h}+\beta \omega_{h}\right]+e^{-a}\left[u_{l}+\beta \omega_{l}\right]-a \\
\text { s.t. } 0 \leq u_{i} \leq u\left(\theta_{i} k^{\alpha}-\delta k\right), \quad \forall i=h, l, \\
\underline{\omega} \leq \omega_{i} \leq \omega \forall i=h, l .
\end{gathered}
$$

In turn, this means that as long as effort is strictly positive, $\bar{\omega}$ satisfies

$$
\begin{aligned}
\bar{\omega}=\max _{s \geq 1, u_{h}, \omega_{h}} & u_{h}+\beta \omega_{h}-1-\log (s), \\
\text { s.t. } & 0 \leq u_{i} \leq u\left(\theta_{i} k^{\alpha}-\delta k\right), \quad \forall i=h, l, \\
& \underline{\omega} \leq \omega_{i} \leq \bar{\omega} \quad \forall i=h, l .
\end{aligned}
$$

The solution of the optimization problem requires that current and continuation utilities are set equal to their upper bounds. That is, $\omega_{i}(\bar{\omega})=\bar{\omega}$ and $u_{i}(\bar{\omega})=\bar{u}_{i}$ for $i=h, l$. Furthermore, $\bar{\omega}=\frac{1}{1-\beta}\left[\bar{u}_{h}-1-\log \left(\bar{u}_{h}-\bar{u}_{l}\right)\right]$ and $v(\bar{\omega})=0$.

Unfortunately, standard sufficient conditions for concavity of the value function do not hold in this scenario, as the feasible set is not convex. However, in all our 

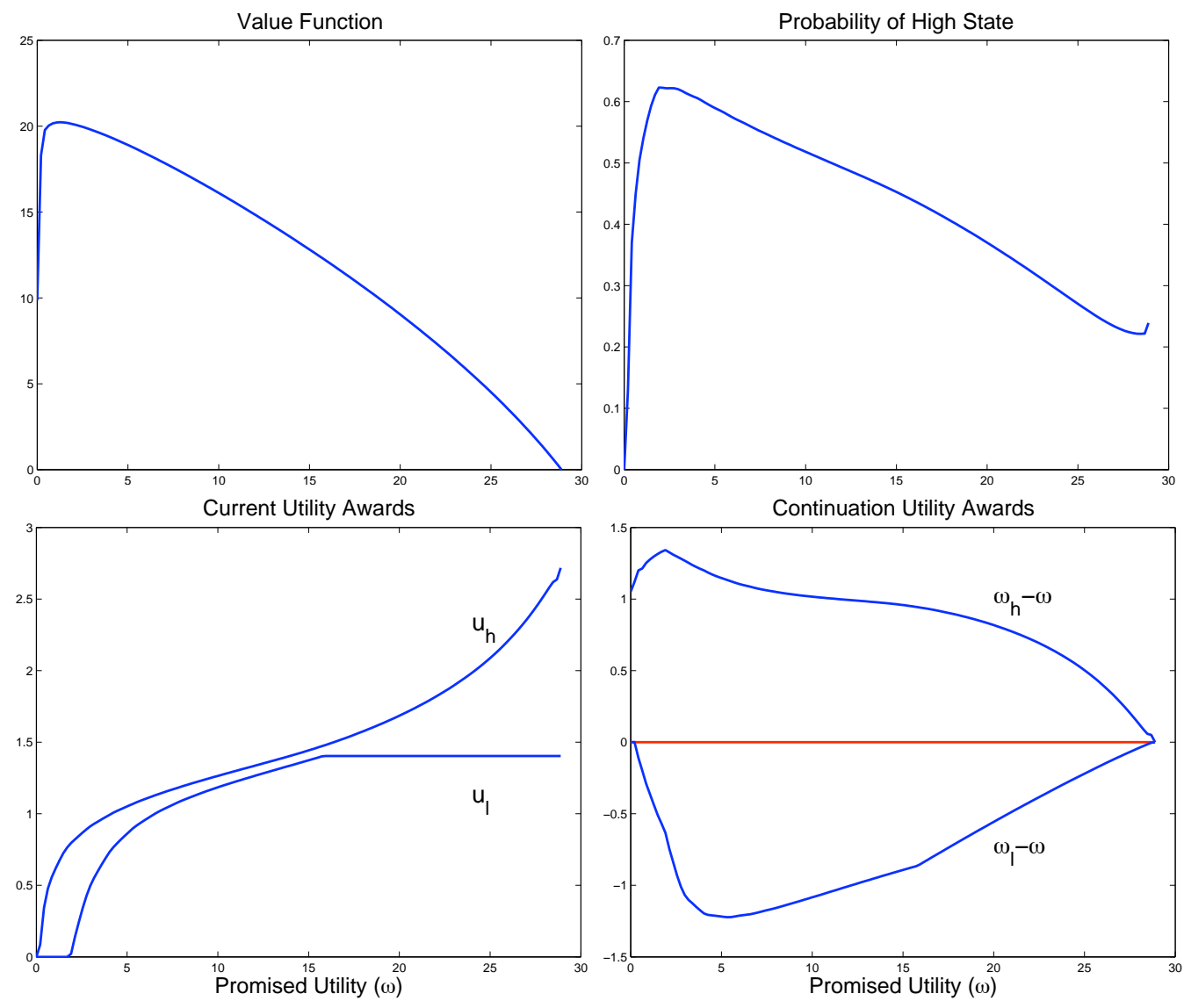

Figure 3: Value and Policy Functions for $\chi=1 / 2$ and $\bar{u}_{h}-\bar{u}_{l}>1$.

numerical examples, the value function turned out to be strictly concave. Figure 3 refers to one such example, for $\chi=1 / 2$ and $\bar{u}_{h}-\bar{u}_{l}>1$.

Consistent with the above discussion, effort is increasing for low $\omega$. This is why the value function is increasing in this range, implying that the contract is not renegotiation-proof. Once $\omega$ is such that current utility is greater than its lower bound, recommended effort and the spread between current utility awards decline with $\omega$. Finally, for relatively high levels of the state variable, the limited liability constraint binds in the bad state of nature. Given the upper bound on continuation utility $\omega$, implementing higher $\omega$ necessarily requires increasing the spread between current utilities.

The dynamical system has two absorbing points, for $\omega=0$ and $\omega=\bar{\omega}$. However, all of our simulations indicate that the latter is the only attractor. For all strictly positive initial conditions, the sequence $\left\{\omega_{t}\right\}$ converges to $\bar{\omega}$ almost surely. On average, 
the entrepreneur's consumption increases over time, converging to its rest point from below. Effort increases on average when the promised utility is relatively low and decreases when it is high.

When the contract is renegotiation-proof, consumption and leisure are complementary ways of rewarding the entrepreneur. High effort is elicited at the cost of low insurance. In such condition, delivering utility via consumption is particularly expensive for the investor. High average levels of compensation are more efficiently delivered when recommended effort is low and the entrepreneur bears relatively less risk.

This simple mechanism also characterizes the scenario with $\chi>1$. Numerical simulations show that effort increases when consumption drops, and vice-versa. The limiting behavior, however, is different.

For $\chi>1$, utility is unbounded below. In turn, this means that all values $\omega \leq \bar{\omega}<0$ are implementable. When the RRA coefficient is greater than 1 , condition (21) implies that payments to the entrepreneur follow a super-martingale, and therefore decrease on average. These considerations suggest that the contract should be front-loaded. High payments and low effort early on, followed by a declining pattern for consumption and a rise in effort. Our inability to characterize analytically the comparative statics of effort also prevents us from formally establishing this result. Not even a numerical verification of this conjecture is not possible, because in order to compute the constrained-efficient allocation, it is necessary to impose an arbitrary lower bound on promised utility $\underline{\omega}$. Under such condition, the limiting distributions for all relevant variables are non-degenerate.

By lowering the elasticity of substitution, a higher level of $\chi$ diminishes the entrepreneur's tolerance for postponing consumption. This simple mechanism is likely at the origin of the differences between the scenarios with $\chi<1$ and $\chi>1$, respectively.

\section{The Full-fledged Model with Capital Accumulation}

We now turn to the general case with capital accumulation. Once again, we focus on the scenario where $\chi<1$. The case of $\chi>1$ will be considered in Section 4.2.

The left panel of Figure 4 depicts $\Omega$, the set of promised utilities that can be delivered by a feasible and incentive-compatible contract. The lower-contour of $\omega$, denoted as $\underline{\omega}$, was chosen arbitrarily, making sure that the contract is renegotiationproof. The upper contour $\bar{\omega}(k)$ is the fixed point of the APS operator $B$, defined 


$$
\begin{gathered}
B(\omega)(k) \equiv \max _{a \in A, k^{\prime},\left\{u_{i}, \omega_{i}\right\}_{i=h, l}}\left(1-e^{-a}\right)\left[u_{h}+\beta \omega_{h}\right]+e^{-a}\left[u_{l}+\beta \omega_{l}\right]-a \\
\text { s.t. } 0 \leq u_{i} \leq u\left[\theta_{i} k^{\alpha}+(1-\delta) k-k^{\prime}\right], \quad \forall i=h, l, \\
\underline{\omega} \leq \omega_{i} \leq \omega\left(k^{\prime}\right) \forall i=h, l .
\end{gathered}
$$

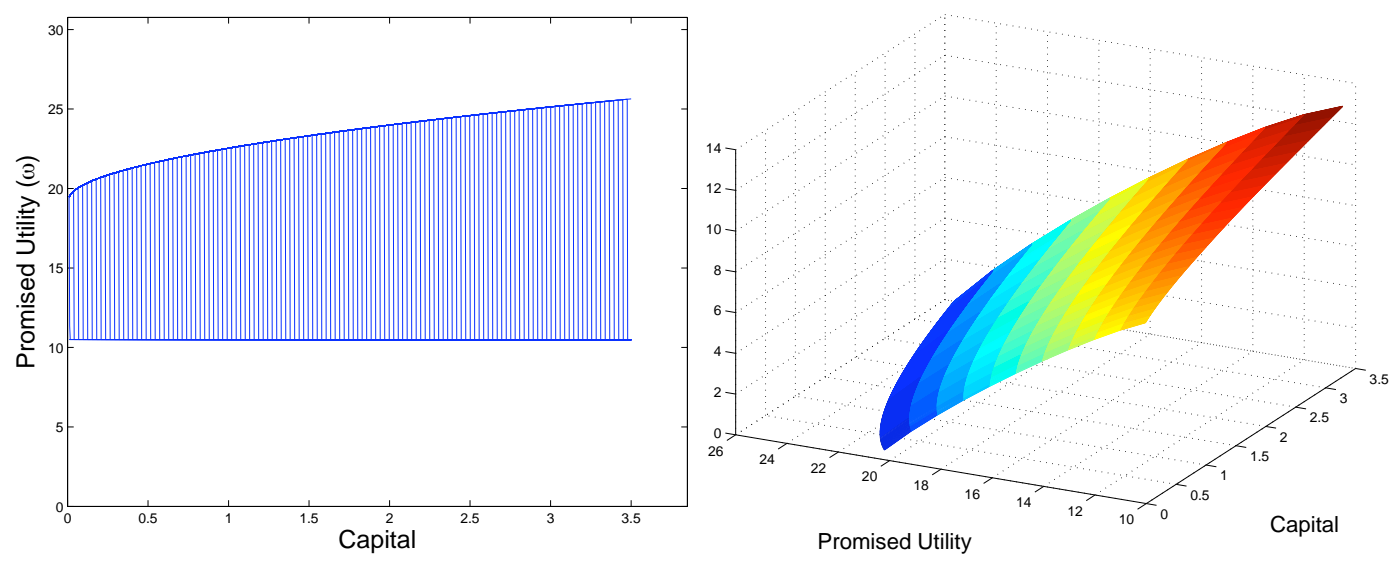

Figure 4: Set $\Omega$ and Value Function.

As expected, $\bar{\omega}(k)$ is increasing in $k$. The solution to (P3) implies that, for every pair $(k, \omega)$ such that $\omega=\bar{\omega}(k)$, the optimal contract dictates $u_{i}(k, \omega)=u\left[\theta_{i} k^{\alpha}+(1-\right.$ $\left.\delta) k-k^{\prime}\right], \omega_{i}(k, \omega)=\bar{\omega}\left(k^{\prime}\right)$ for $i=h, l$, and $v(k, \omega)=0$.

The right panel shows that the value function is strictly increasing in the level of capital and strictly decreasing in the entrepreneur's promised utility $\omega$. In all of our numerical examples, $v(k, \omega)$ is also globally strictly concave. However, since standard sufficient conditions for concavity are not satisfied, we cannot assert this as a general property.

Figure 5 depicts the policy functions for current and promised utility. In the left panel, we have plotted $u_{i}(k, \omega), i=h, l$. In the right panel, we have pictured the contingent variation in promised utility $\omega_{i}(k, \omega)-\omega, i=h, l$.

For given capital stock, the entrepreneur's contingent compensation schedules display the same qualitative features as in the case with no accumulation. The spread between continuation utilities appears to be decreasing in $\omega$, while the spread between current utilities is decreasing in $\omega$ for low values and increasing for high values. Once again, this is due to the fact that the limited liability constraint binds in the bad state of nature. 

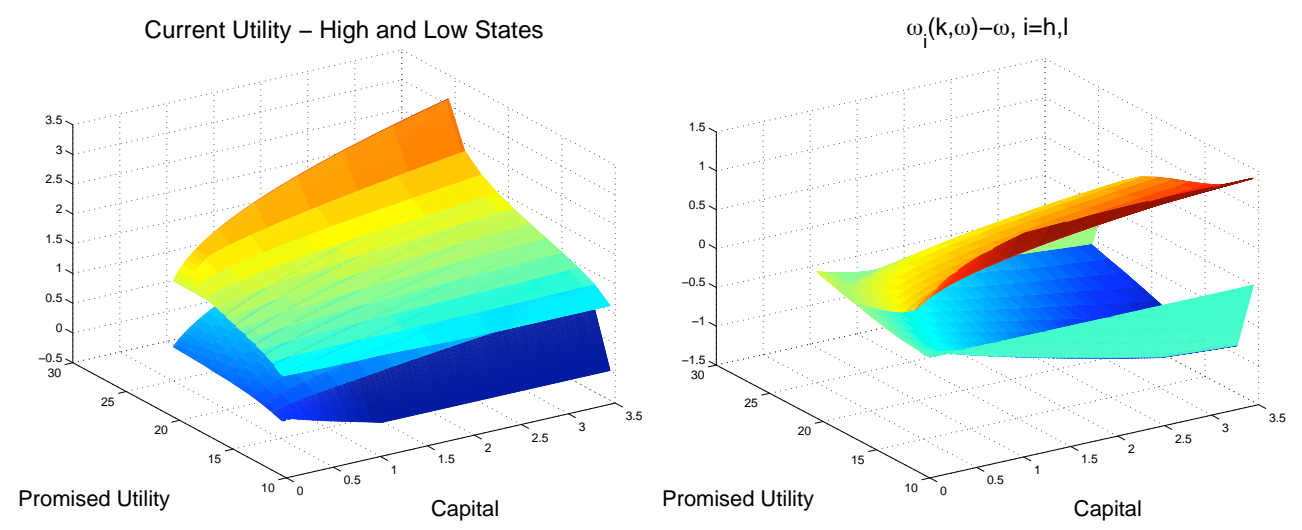

Figure 5: Compensation Policies.

The necessary condition for optimality of the effort choice is the analogue of equations (18). Refer to the left panel of Figure 6. Consistent with the intuition developed in Section 3, recommended effort is increasing in the capital stock and decreasing in the level of promised utility $\omega$.
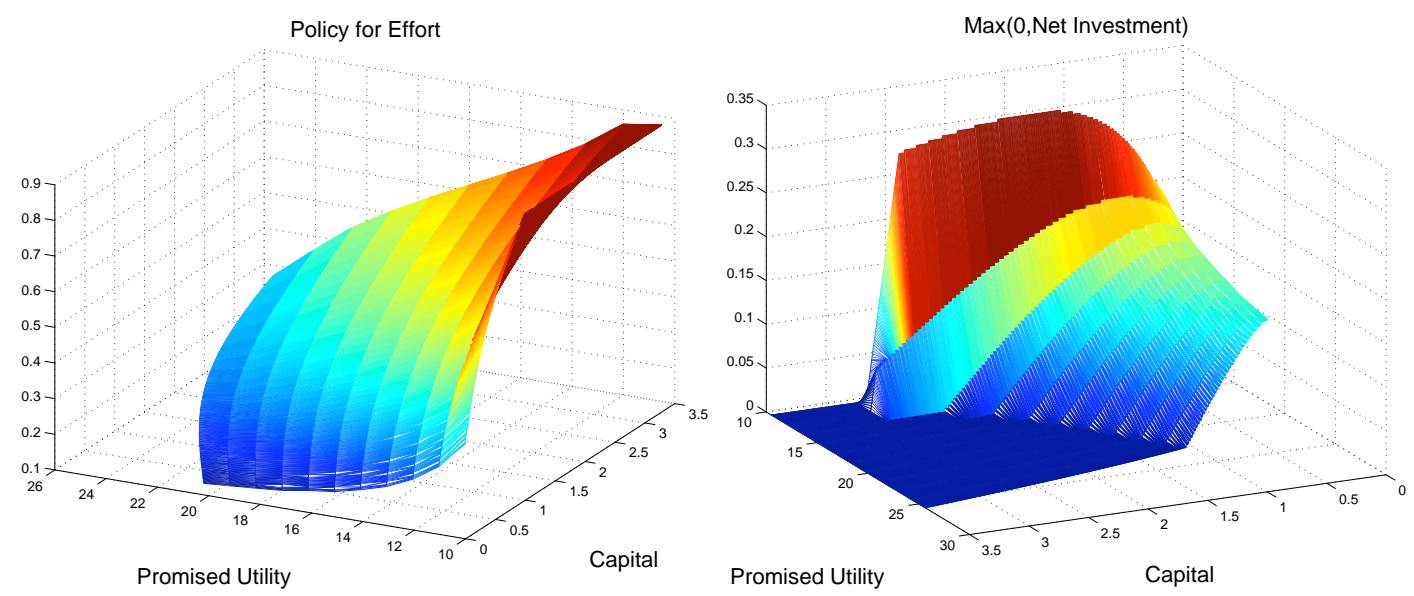

Figure 6: Policy Functions for Effort and Net Investment.

The novelty with respect to the simpler models analyzed above is capital accumulation. The policy function for net investment is rendered in the right panel of Figure 6 , where we plotted $\max \left[0, k^{\prime}(k, \omega)-k\right]$. The most interesting feature is that, for given capital, net investment is declining in $\omega$. The optimality conditions tell us why this is the case.

Consider first the scenario in which the limited liability constraint is slack. When 
the value function is differentiable, the first--order condition for the capital choice is

$$
\left(1-\frac{1}{s}\right) \frac{\partial v\left(k^{\prime}, \omega_{h}\right)}{\partial k^{\prime}}+\frac{1}{s} \frac{\partial v\left(k^{\prime}, \omega_{l}\right)}{\partial k^{\prime}}=\frac{1}{\beta} .
$$

From the envelope theorem, it follows that

$$
\frac{\partial v(k, \omega)}{\partial k}=\left[\left(1-\frac{1}{s}\right) \theta_{h}+\frac{1}{s} \theta_{l}\right] \alpha k^{\alpha-1}+(1-\delta) .
$$

Differentiating the latter with respect to $\omega$ yields

$$
\frac{\partial^{2} v(k, \omega)}{\partial k \partial \omega}=\frac{1}{s^{2}}\left(\theta_{h}-\theta_{l}\right) \alpha k^{\alpha-1} \frac{\partial s}{\partial \omega} .
$$

Since $\partial s / \partial \omega<0,(23)$ says that the marginal effect on future net cash-flows deriving from an increase in capital is decreasing in $\omega$. The higher $\omega$, the lower the optimal utility spread $s$ and the probability of success. In turn, this leads to a lower marginal value of investment.

Now fix $k$ and consider the effect of increasing $\omega$ on the optimal choice of $k^{\prime}$. As long as the value function is concave, such effect will have the same sign as that of the derivative of (22) with respect to $\omega$. The first term, $\frac{1}{s^{2}}\left[\frac{\partial v\left(k^{\prime}, \omega_{h}\right)}{\partial k^{\prime}}-\frac{\partial v\left(k^{\prime}, \omega_{l}\right)}{\partial k^{\prime}}\right] \frac{\partial s}{\partial \omega}$, is positive. A higher value of $\omega$ today leads to a lower utility spread $s$, which in turn increases the probability of a bad outcome. Since $\omega_{h}>\omega_{l}$, this means that the effect on the marginal gain is positive. On the other hand, as long as $\omega_{h}$ and $\omega_{l}$ are strictly increasing in $\omega$, the second term $\left(1-\frac{1}{s}\right) \frac{\partial^{2} v\left(k^{\prime}, \omega_{h}\right)}{\partial k^{\prime} \partial \omega_{h}} \frac{\partial \omega_{h}}{\partial \omega}+\frac{1}{s} \frac{\partial^{2} v\left(k^{\prime}, \omega_{l}\right)}{\partial k^{\prime} \partial \omega_{l}} \frac{\partial \omega_{l}}{\partial \omega}$ is negative. In our simulations, the latter effect dominates. Investment decreases with $\omega$.

When the limited liability constraint binds in the bad state of nature, the optimality condition for capital reads as follows:

$$
\left(1-\frac{1}{s}\right)\left[\beta \frac{\partial v\left(k^{\prime}, \omega_{h}\right)}{\partial k^{\prime}}-1\right]+\frac{1}{s}\left[\beta \frac{\partial v\left(k^{\prime}, \omega_{l}\right)}{\partial k^{\prime}}+\left(c^{\prime}\left(u_{l}\right)+\frac{\partial v\left(k^{\prime}, \omega_{l}\right)}{\partial \omega_{l}}\right) u^{\prime}\left(c_{l}\right)\right]=0 .
$$

with $c_{l}=\theta_{l} k^{\alpha}+(1-\delta) k-k^{\prime}$ and $u_{l}=u\left(c_{l}\right)$. The term $c^{\prime}\left(u_{l}\right)+\frac{\partial v\left(k^{\prime}, \omega_{l}\right)}{\partial \omega_{l}}$ is the marginal effect of increasing $\omega_{l}$. Since the limited liability constraint binds, it must be negative. An increase in $k^{\prime}$ distorts compensation in the bad state of nature, leading to a drop in $u_{l}$ to the advantage of $\omega_{l}$. In other words, an increase in $k^{\prime}$ lowers the investor's payoff by reducing the insurance the contract provides to the entrepreneur. Unfortunately the comparative statics of $k^{\prime}$ is now considerably more involved. The role of the limited liability constraint in shaping firm dynamics will be re examined in Section 4.1.

Figure 7 illustrates one simulation of the system, starting from arbitrary initial conditions. The state space is partitioned in four subsets. The policy for capital is 


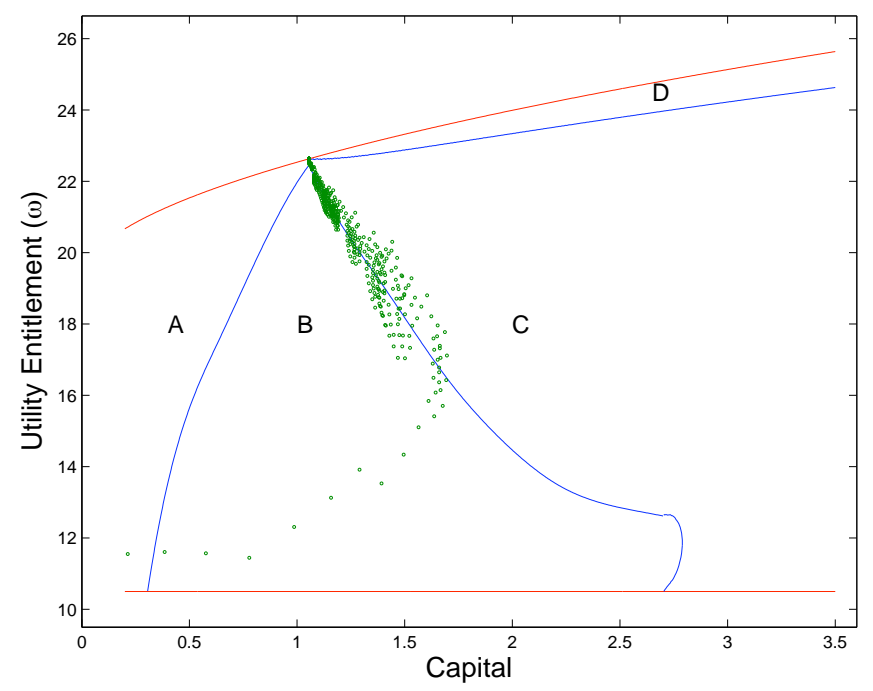

Figure 7: Sample Path.

such that $k^{\prime}(k, \omega)>k$ in regions $\mathrm{A}$ and $\mathrm{B}$, and $k^{\prime}(k, \omega)<k$ otherwise. The dynamics of promised utility is such that in regions B and C, $\omega_{h}(k, \omega)>\omega>\omega_{l}(k, \omega)$. Irrespective of the realization of the shock, promised utility increases in region A and decreases in region $\mathrm{D}$, respectively. In light of the above discussion, it is not surprising that the locus separating regions $\mathrm{B}$ and $\mathrm{C}$, along which net investment is identically zero, is downward sloping for most values of $k$.

The scatter plot illustrates the dynamics of the state variables. The paths followed by the other relevant variables are shown in Figure 8. When $k$ and $\omega$ are relatively low, the marginal product of capital is high and providing incentives is relatively inexpensive. Therefore the returns to investment are high. Capital grows in both states of nature. During the transition towards the locus separating the partitions B an $\mathrm{C}$, all other variables also increase on average. However, increases in both $\omega$ and $k$ lead to a progressive reduction in the marginal gain from capital accumulation. Once reached the locus, incentive provision becomes so expensive to discourage investment. From that moment onwards, positive shocks lead to contemporaneous increases in current and future payouts to the entrepreneur, and to a decrease in the continuation value of the investor's claim. The next period, the capital and effort choice will be lower. The opposite will be true, conditional on negative shocks. ${ }^{9}$

\footnotetext{
${ }^{9}$ Changing the timing in such a way that capital is chosen after the realization of the shock would definitely enhance efficiency, but it would not have a sizeable impact on dynamics. The main difference would be that capital reacts to shock realizations with one period delay, rather than two periods, as is the case under our assumptions.
} 

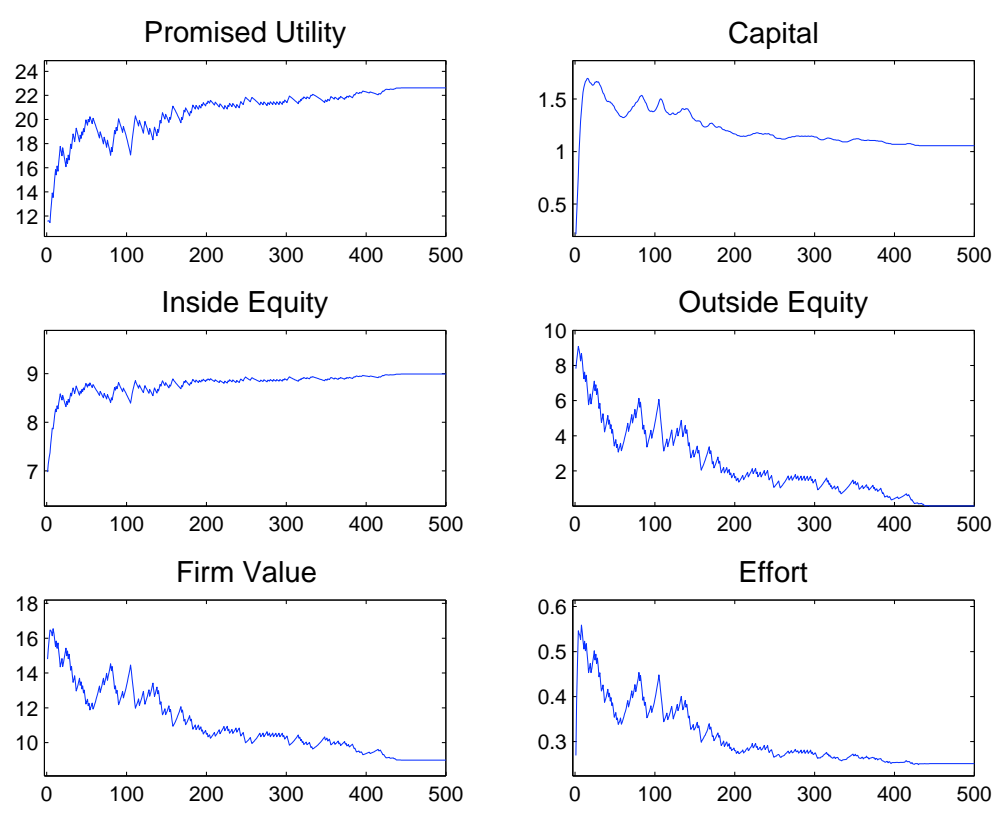

Figure 8: Sample Path.

On average, $\omega$ increases over time. This calls for lower effort, lower capital, and lower outside equity. Eventually the system converges to a steady state where outside equity is zero and the entrepreneur's promised utility lies on the upper contour of the set $\Omega$. The constrained-efficient arrangement prescribes that in the limit the entrepreneur controls all cash-flow rights.

Notice further that, even at the steady state, the contract does not yield full insurance and requires strictly positve effort. This result does not hold with generality. When the difference between $\theta_{h}$ and $\theta_{l}$ is small enough, the entrepreneur will end up receiving full insurance and exerting no effort.

Staring at Figure 9 confirms what we have learned so far. We have initialized the system by assigning to the state variables the same initial conditions used to construct Figures 7 and 8. Then we have conducted a large number of 80-period long simulations and plotted their simple averages. Early on all variables tend to increase. Eventually, however, effort, capital, and the value of the investor's claim start declining.

We have assumed throughout that no other agent is able to operate the technology. Or, alternatively, that the investor is fully committed to the contract. It would be interesting to understand under what conditions the investor would be better off by rescinding the contract, honor his promises by means of a constant sequence of 

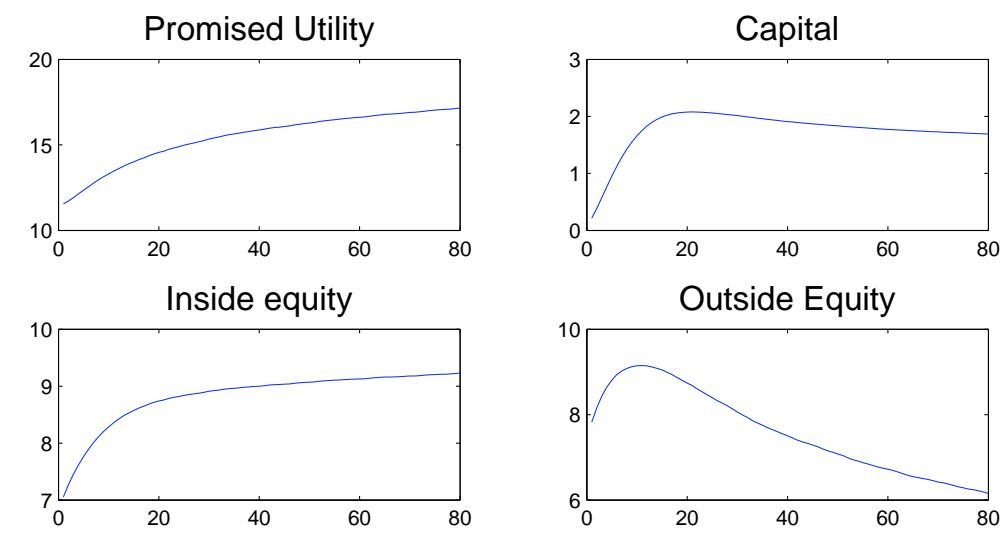

Outside Equity

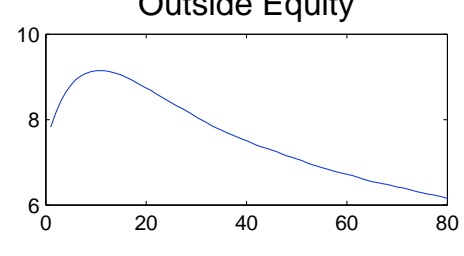

Firm Value
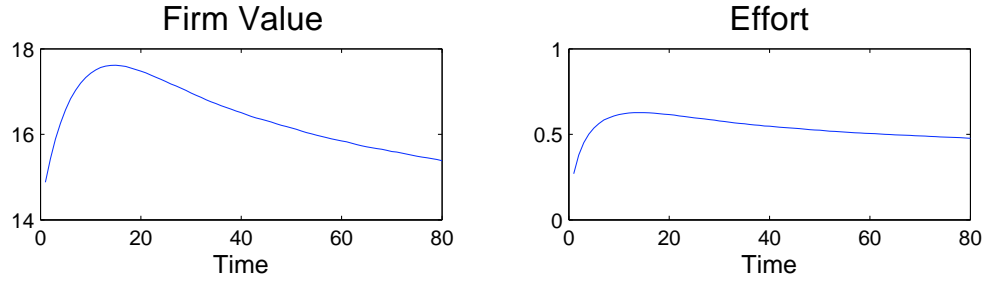

Figure 9: Average Dynamics.

consumption, and hire someone else to manage the project. Spear and Wang (2005) and Wang (2006) address this issue in simpler environments, without production or capital accumulation.

\subsection{The Role of the Limited Liability Constraint}

The purpose of this section is to clarify how the constrained-efficient allocation is affected by the limited liability constraint. We'll accomplish this task by discussing the allocation that obtains when the constraint is not imposed and $\chi<1$.

Without upper bounds to entrepreneur's consumption, all non-negative levels of utility could be delivered by a feasible and incentive compatible contract. For the purpose of computations, we simply impose an exogenous upper bound. ${ }^{10}$

A first and somewhat obvious finding is that, given $\omega$ and no matter the initial capital $k_{1}$, it is optimal for the investor to invest (or disinvest) instantaneously until the firm lies on the locus separating the partitions B and C in the state space (see Figure 7).

Our numerical experiments also show that, as it was the case in our benchmark

\footnotetext{
${ }^{10}$ The simulation of which in Figure 10 was obtained by imposing $\omega_{i}(k, \omega) \leq 50$ for $i=h, l$ and for all $(k, \omega)$. However, the qualitative features illustrated below are the same, no matter the upper bound.
} 

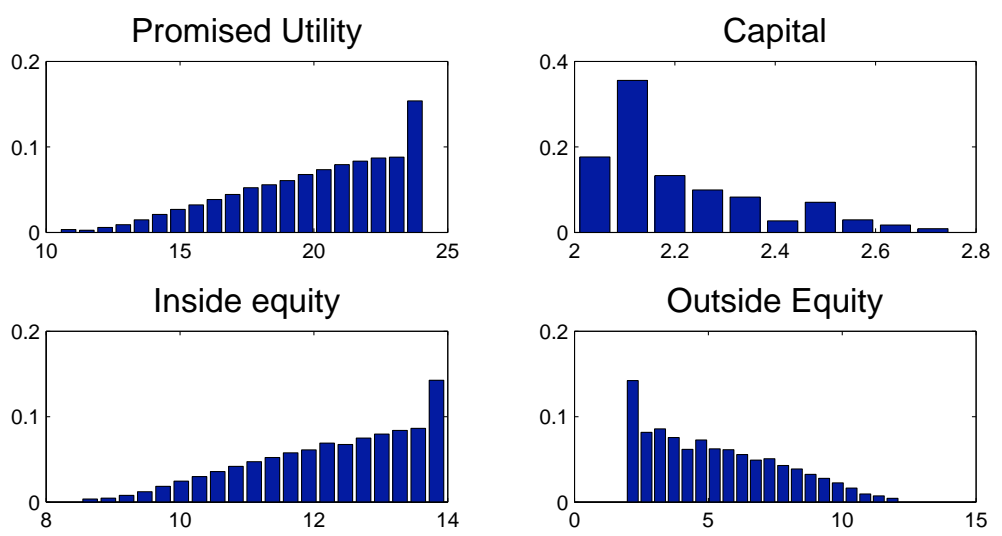

Firm Value

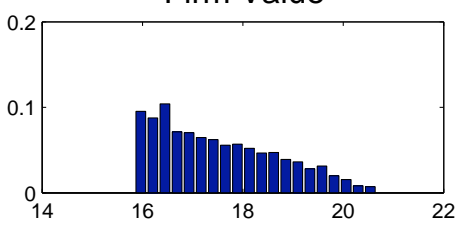

Effort

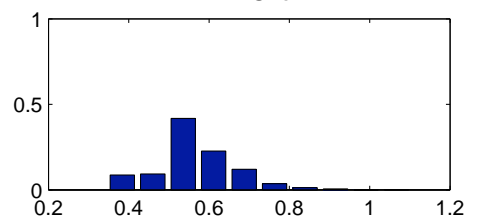

Figure 10: Stationary Distribution - Without Limited Liability.

scenario, positive shocks are followed by an increase in promised utility in the same period and by lower capital and lower effort in the following period. However, the limiting behavior is different. The dynamical system admits stationary distributions for all relevant variables, illustrated in Figure $10{ }^{11}$

As long as ventures start out with a relatively low level of inside equity (i.e. low $\omega)$, firm value, outside equity, size, and effort will decrease over time. Inside equity will increase. The fact that firm value and size are maximal at the beginning of the life-cycle is at odds with the empirical evidence. Requiring that investment is financed by retained earnings, the limited liability constraint avoids this unappealing feature. The reader that feels uncomfortable with such assumption should consider that convex capital adjustment costs of the type commonly assumed in the macroeconomic literature would lead to a dynamics which is qualitatively the same as in our benchmark case. In particular, for a low enough initial capital stock, net investment would be positive early on and would decrease (on average) later on.

\footnotetext{
${ }^{11}$ The figure was obtained initializing the system with arbitrary levels of the state variables and letting the system run for 50,000 period. We subtracted the first 500 and reported the frequency distributions.
} 


\subsection{The case of $\chi>1$}

The basic theme of this Section also applies to this scenario: positive shocks are associated to an increase in promised utility in the same period and by lower capital and lower effort in the following period. However, differently from the case of $\chi<1$, the dynamical system admits stationary limiting distributions for all relevant variables. Figure 11 illustrates such distributions when $\chi=2$ and the lower bound on promised utility is $\underline{\omega}=-100$.
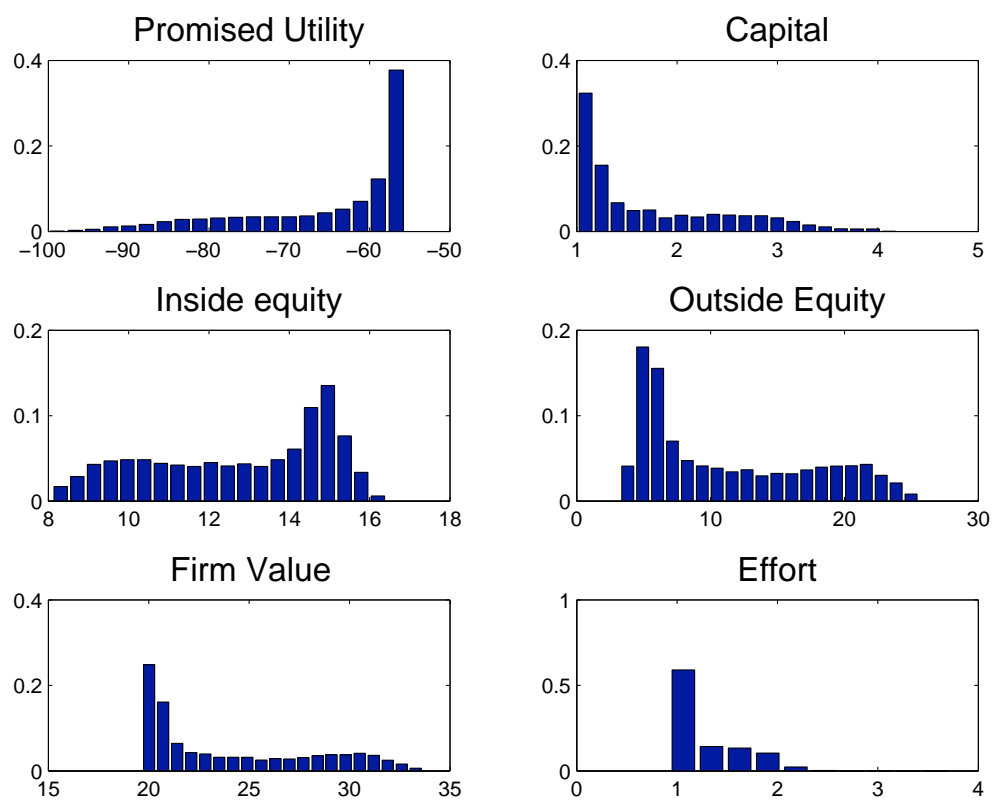

Figure 11: Stationary Distribution for $\chi=2$.

When the system is initialized with relatively low levels for capital and promised utilities, average dynamics is qualitatively similar to that implied by our benchmark. In particular, capital, outside equity, and firm value increase early on, and then decrease. On average, inside equity and payments to the entrepreneur are monotonically increasing.

In light of the fact that for $\chi>1$, condition (21) implies that consumption is a super-martingale, the result just illustrated may sound surprising. Notice however that (21) holds only when the solution is interior. In fact, inspection reveals that when the limited liability constraint binds, expected consumption growth following a bad shock is positive. 


\section{Empirical Relevance}

Much of the empirical literature on firm dynamics has focused on growth and survival of relatively young firms. ${ }^{12}$ Among its most robust findings are that both exit hazard rates and survivors' growth rates decline with age, both unconditionally and conditional on size. Models based on learning or financing constraints have proven successful in rationalizing both phenomena. ${ }^{13}$

As put by Caves (1998), however, "organizational geriatrics has received little attention." That is, little attention has been devoted to the dynamics of relatively older firms. Notable exceptions are the studies conducted by Aggarwal and Gort (1996, 2002) (AG hereafter) and Loderer, Neusser, and Waelchli (2009). Consistent with most of the literature, they find that hazard rates decrease with age early in life. However, they also find that firms eventually reach a senility point, after which exit hazard rates increase with age. In this Section we argue that, differently from other theories of firm dynamics, our own is able to rationalize this evidence.

Exit hazard rates are likely to be the result of both firm-specific and industryspecific attributes. In order to discriminate among the two, AG document firm survival in five different phases of the industry life-cycle. ${ }^{14}$ Aggarwal and Gort (1996) shows that the senility point exists no matter the phase in which firms are born. Aggarwal and Gort (2002) argues that it exists no matter the phase in which firms live. This evidence leads us to conclude that for every cohort there exists a time after which, conditional on industry-wide factors, the value of the average survivor declines with age.

None of the models of firm dynamics we referred to above are consistent with this behavior. All of them predict that survivors' size and value converge from below to a stationary distribution. Exit hazard rates are monotonically decreasing and converge to a constant.

AG posit that the observed firm dynamics depends in part on the evolution of firms' "initial endowments," among which Aggarwal and Gort (1996) include "observed variables, such as an initial organization with a record of successful operation in a related industry," and "unobserved variables such as managerial talent." In every

\footnotetext{
${ }^{12}$ See Caves (1998) for a survey.

${ }^{13}$ See Jovanovic (1982) in the case of learning and Albuquerque and Hopenhayn (2004), Clementi and Hopenhayn (2006), Cooley and Quadrini (2001) and Quadrini (2003) in the case of financing constraints.

${ }^{14}$ The five phases are supposed to track the industry in its path from infancy to maturity. AG identify them operationally by means of Bahk and Gort (1993)'s criterion, based on the rate of net entry.
} 
cohort the average endowment level changes over time because of attrition of low endowment firms and because of survivors' net investment. As firms age, obsolescence rises with respect to new investment. Eventually, net investment will turn negative and lead firms to decline. According to AG, this is the mechanism responsible for the fact that, for relatively old firms, hazard rates increase with age.

AG's argument has the flavor of the simple technological theory of industry dynamics presented in Hopenhayn (1992). In that paper, a firm's endowment is a stationary and serially correlated random variable determining productivity. The average entrant has a lower endowment than the average incumbent, and is therefore closer to the exit threshold. This assumption is responsible for the selection effect that drives the negative correlation between age and hazard rate. Because of the stationarity of productivity, every cohort's size distribution converges to an ergodic distribution (also stationary, when re-scaled by its mass), characterized by a constant exit rate. In turn, this means that the hazard exit rate also converges to a constant from above. Differently from what advocated by AG, on average obsolescence of the endowment is always smaller than new investment.

In our model, AG's endowment is managerial effort, which in turn is responsible for the productivity of the capital invested. The analysis conducted in Section 4 suggests that almost surely there exists a point in time after which the endowment is expected to decline. Think of a cohort of firms whose dynamics are generated by our contract. Optimal incentive provision prescribes that, on average, promised utility increases over time. In turn, this calls for lower effort and lower capital, which imply lower value. While we do not explicitly model exit, it is clear that assuming a constant outside value for the assets would yield a positive relation between age and exit hazard rate.

We conclude this section by speculating on our framework's predictions for the cross-sectional relationship between personal wealth and entrepreneurship. Newman (2007) shows that a static version of our model has a patently counterfactual implication. When individuals in a population are given the choice between working for a wage (at zero risk) and becoming entrepreneurs, relatively rich people turn out to be workers, and poor people become entrepreneurs. As long as the value assigned to the agent by the contract is monotone in the agent's wealth, it is easy to see why this is the case. Everything else equal, the poorer the entrepreneur the cheaper is incentive provision, and the larger is the payoff to investors. Our analysis shows that in general Newman (2007)'s conclusion is not warranted in a dynamic setting. While it is still 
true that poorer people are more likely to try entrepreneurship, it is also true that those among them that succeed become progressively wealthier.

\section{Comparative Statics}

In this section, we document how the optimal contract and the implied dynamics change when we select alternative values for either the entrepreneur's discount factor or the support of the conditional distribution $\Theta$.

\subsection{An Impatient Entrepreneur}

Throughout Sections 3 and 4 it was assumed that the two agents discount future utility flows at the same rate. Here we consider the case in which the entrepreneur discounts future utility at the rate $\frac{1}{\rho}-1$, with $\rho<\beta$.

Figure 12 compares the policy functions that obtain for $\rho=0.495$ with those relative to the benchmark case. Given the entrepreneur's preference for early consumption, the optimal contract calls for a change in the time profile of her cash flows in favor of the current period. The right panel on the bottom row shows that, everything else equal, the entrepreneur receives higher payments in both states of nature.
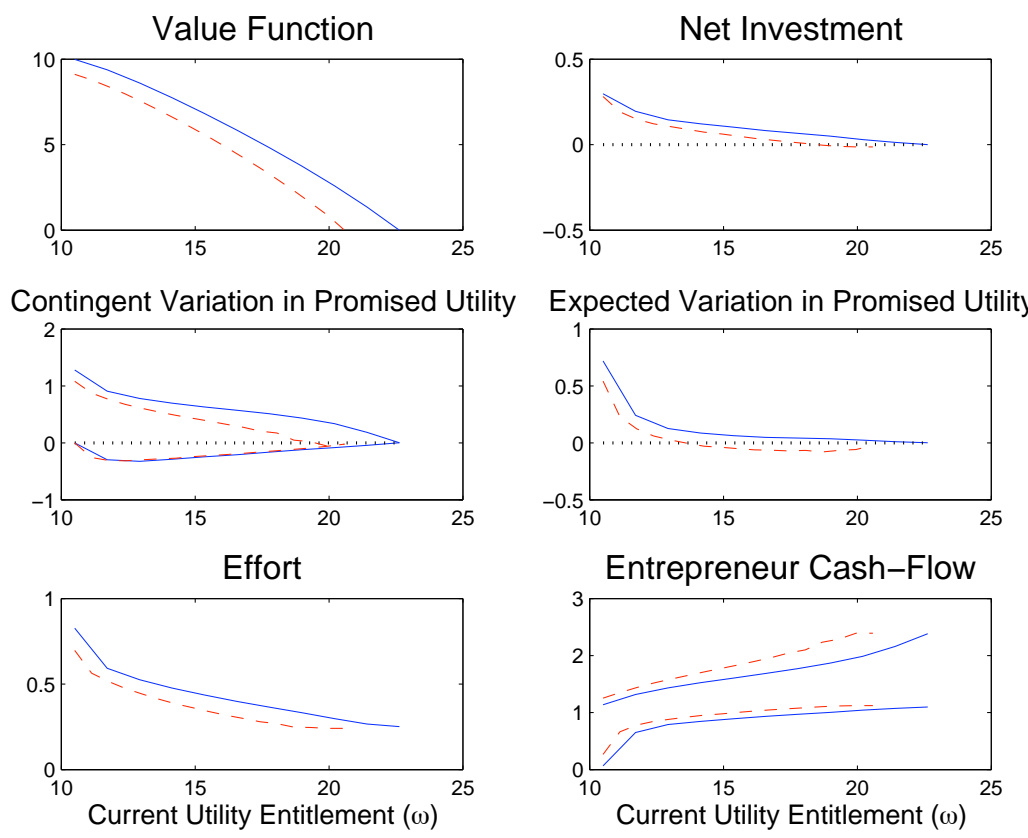

Figure 12: Policy Functions. Solid: benchmark. Dashed: $\rho=0.945$ 
When the limited liability constraint binds, i.e. when $u_{l}=u\left[\theta_{l} k^{\alpha}+(1-\delta) k-\right.$ $\left.k^{\prime}\right]$, a higher payment to the entrepreneur necessarily translates into fewer resources available for investment. This is why, as illustrated on the right panel on the top row, net investment is lower than in the benchmark. The left panel on the bottom row shows that recommended effort drops. This is consistent with our discussions in Sections 3 and 4: a rise in current utility awards also raises the marginal cost of eliciting effort.

Our simulations show that, as in the case of $\chi>1$, capital, promised utility, as well as the other relevant variables converge to non-degenerate stationary distributions.

\subsection{A Narrower Gap between $\theta_{h}$ and $\theta_{l}$.}

Since the probability of good outcome is endogenous in our model, so is the distribution of cash-flows. Therefore, the closer we can get to analyzing the role of cash-flow risk, is to consider the impact of reducing the gap between the two realizations of the productivity shock.

Figure 13 plots the policy functions that obtain by simultaneously lowering $\theta_{h}$ from 1.5 to 1.45 and raising $\theta_{l}$ from 0.4 to 0.45 . Everything else equal, a lower gap
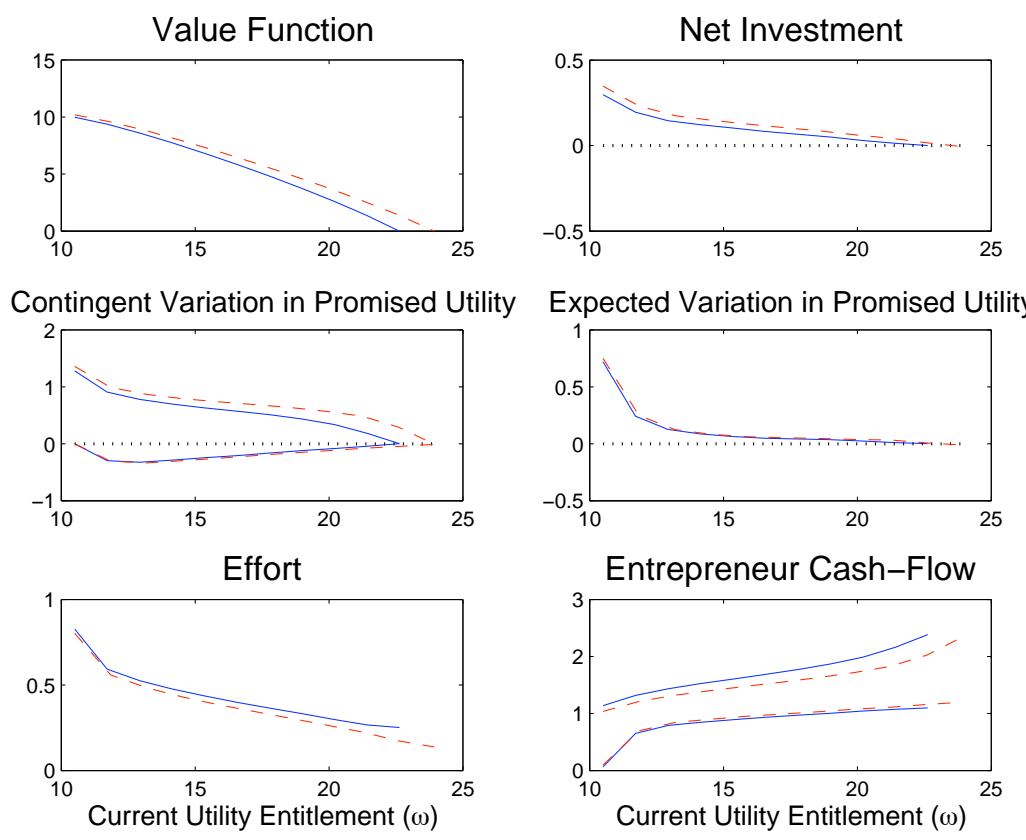

Figure 13: Policy Functions. Solid: benchmark. Dashed: $\theta_{h}=1.45, \theta_{l}=0.45$.

reduces the marginal benefit of effort. This is why the latter drops. In turn, lower 
effort means more insurance for the entrepreneur.

The right panel on the top row shows that investment is higher. Most likely, this result depends on the fact that a higher $\theta_{l}$ relaxes the limited liability constraint in the bad state of nature. As usual, investment is chosen to equate the marginal effect on future revenues to the opportunity cost. In Section 4 we have argued that when the constraint binds, this cost also depends on the intertemporal distortion that is caused by lower consumption in the low state. This is why efficiency dictates that the larger revenues in the low state are allocated in part to increase compensation, as argued above, and in part to increase investment.

\section{Auto-Correlated Shocks}

So far we have assumed that a successful performance of the entrepreneur only affects the probability distribution of the shock $\theta$ in the same period. Although it is shared by most of the literature on dynamic hidden action models, ${ }^{15}$ one may find this assumption to be particularly removed from reality. In this section we address this concern by assuming that a successful outcome also alters the probability distribution in the future. We posit that next period's distribution conditional on success in the current period, $G\left(\theta^{\prime} \mid a^{\prime}, \theta_{h}\right)$, stochastically dominates the distribution conditional on failure, i.e. $G\left(\theta^{\prime} \mid a^{\prime}, \theta_{l}\right)$, for all $a^{\prime} \in A$. In the numerical implementation, we assume that $\operatorname{prob}\left(\theta^{\prime}=\theta_{h} \mid \theta=\theta_{i}\right)=1-e^{-\psi_{i} a}$, with $\psi_{h}>\psi_{l}>0$.

Notice that our modeling choice is different from that of Fernandes and Phelan (2000). In their case, next period's distribution depends on current effort (which is private information), rather than on the current realization (public information).

Figure 14 illustrates value and policy functions along the $\omega$ dimension, for given capital stock. Solid lines refer to $\psi_{h}=1.4$, while dashed line refer to $\psi_{l}=0.8$. All the other parameters are as described in Table 1. For $\psi=\psi_{h}$, a given level of effort leads to a higher probability of success. With respect to the case of $\psi=\psi_{l}$, the promised utility $\omega$ is delivered by requiring more effort and awarding greater average consumption and expected continuation utility. Given the higher probability of success, this plan can be implemented by lowering the state-contingent payoffs to the entrepreneur, both in the present and in the future. This is why $c_{i}\left(k, \omega, \psi_{l}\right)>$ $c_{i}\left(k, \omega, \psi_{h}\right)$ and $\omega_{i}\left(k, \omega, \psi_{l}\right)>\omega_{i}\left(k, \omega, \psi_{h}\right)$ for all $\omega$ and for $i=h, l$.

In spite of the fact that average continuation utility is higher, net investment is

\footnotetext{
${ }^{15}$ For example, see Spear and Srivastava (1987) and Wang (1997).
} 
higher for $\psi=\psi_{h}$. The reason is simple. The persistence in the distribution of the shock and the difference between $\theta_{h}$ and $\theta_{l}$ are such that the marginal value of investment is higher in that case.
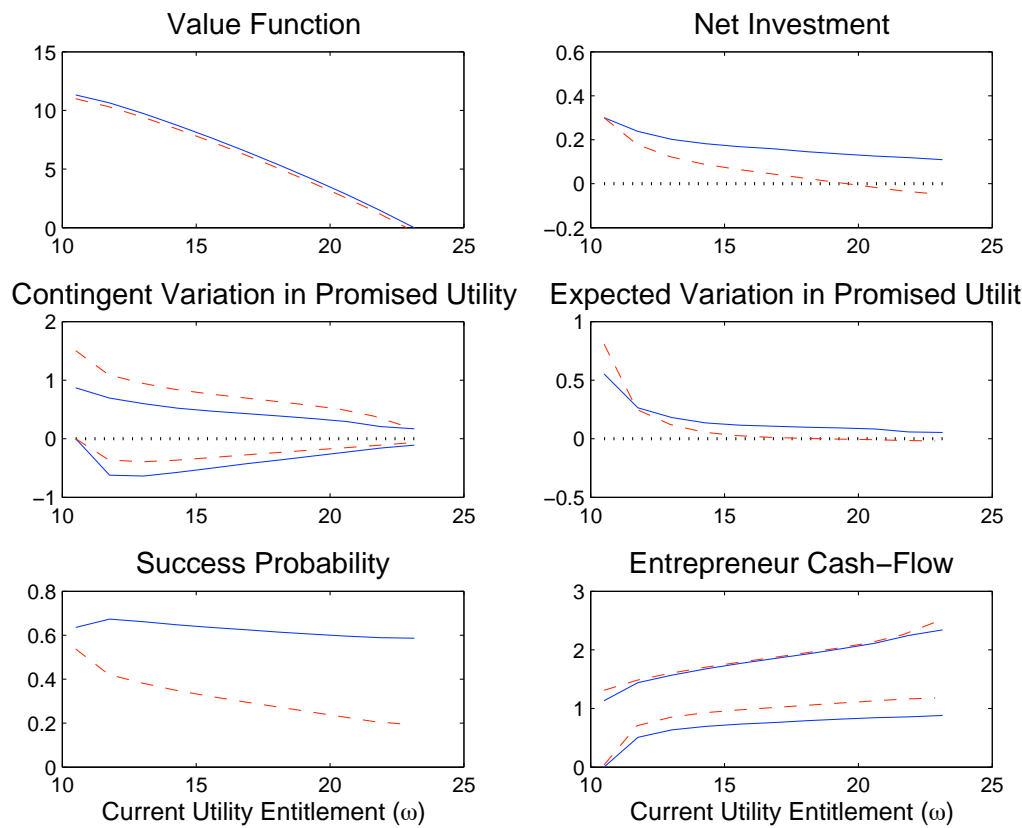

Figure 14: Policy Functions. Solid: $\psi=\psi_{h}$. Dashed: $\psi=\psi_{l}$.

Figure 15 illustrates the effects of persistence on the dynamics of all relevant variables. A good shock is associated with higher consumption and higher continuation utility. However, differently from the benchmark scenario, firm value as well as next period's investment and recommended effort are also higher following a good shock. Why is this the case?

For simplicity, consider the case of a good realization when the distribution is parameterized by $\psi_{l}$. On the one hand, the rise in continuation utility triggered by the shock still calls for lower effort in the future and therefore implies a lower marginal value of investment. On the other hand, the improvement in the shock distribution, by calling for greater effort, raises the marginal value of investment. For our parameter values, the latter effect dominates.

Similarly to the benchmark scenario described in Section 4, the value of outside equity converges to zero and the entrepreneur ends up controlling all cash-flow rights. However, the ergodic set for the state variables is now a non-degenerate subset of $\Omega$ 's upper contour. As the investor's payoff settles down to its long-run value, the other 
variables are time-varying.
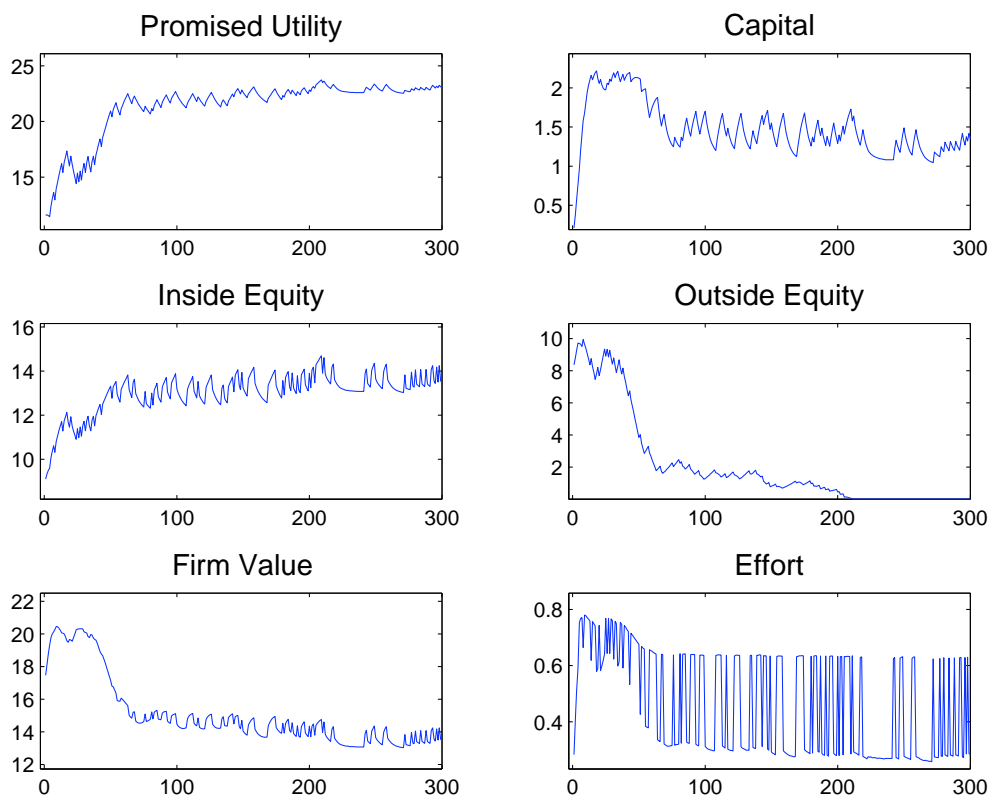

Figure 15: Sample Path. Case with State-Dependent Shock Distribution

\section{Conclusion}

In this paper we have characterized the firm dynamics implied by constrained-efficient contracts between a risk-neutral investor and a risk-averse entrepreneur under the assumption that the latter's effort is not publicly observable.

A robust feature of the model is the sub-modularity of the value function. The marginal gain from investing declines with the level of promised utility. This happens because the cost of incentive provision is increasing in $\omega$. In turn, this means that the higher $\omega$, the lower are recommended effort and the probability of success, and therefore the lower is the return to capital accumulation.

When the entrepreneur's relative risk aversion coefficient is less than 1 and the two agents are equally impatient, $\omega$ and the value of inside equity follow sub-martingale processes. Firms that, consistent with the empirical evidence, start out small, have a relatively high marginal product of capital, and therefore grow over time. The gradualness of the growth process depends on the limited liability assumption, which can be interpreted as a financing constraint or an extreme form of investment adjustment costs. Because of the sub-modularity property, the rise in the value of promised util- 
ity implies a drop in the return to investment, which eventually leads to a decline in the capital stock. In this sense, our theory provides a rationale for firm's decline. This feature distinguishes our model from other theories of firm dynamics. According to those, average firm size and value of survivors increase monotonically over time.

Interestingly, the constrained-efficient contract prescribes that in the long run the entrepreneur becomes the only claimant to the firm's cash flows.

When the RRA coefficient is larger than 1 or the entrepreneur is relatively more impatient, the dynamics of observables has the same qualitative features, as long as the initial values of capital and promised utility are relatively low. What distinguishes these scenarios from our benchmark is that the model admits non-degenerate stationary distributions for firm size and the other relevant variables.

A key mechanism in our theory is that providing incentives to exert effort becomes costlier as the manager increases her stake in the firm. We believe that it would be interesting to extend our framework by allowing for the possibility of termination. That is, by empowering the investor to liquidate the entrepreneur and hire someone else to run operations. The insights provided by Spear and Wang (2005) and Wang (2006) may prove useful in carrying out this task.

We also believe that it would be of interest to study the dynamics implied by our model under different assumptions on preferences. The results by Rampini (2004) hint that under certain conditions the results could be quite different. In his study of the relationship between entrepreneurial activity and the business cycle, he finds that when preferences are of the type $u(c-a)$, risk tolerance is increasing with wealth, and therefore incentive provision is cheaper. Exactly the contrary of what happens in this paper. 


\section{A Proofs and Lemmas}

\section{Proof of Proposition 1.}

Fix the pair $(k, \omega)$. We already know that $\Phi(k, w)$ is bounded. It is left to prove that it is also closed. Let $\left\{V_{n}\right\} \subseteq \Phi(k, w)$, where $V_{n} \rightarrow V_{\infty}$ when $n \rightarrow \infty$. We need to show that $V_{\infty} \in \Phi(k, w)$. In words, we need to demonstrate that there exists a contract $\sigma_{\infty}$ that satisfies (2), (3), (4), $\omega\left(\sigma_{\infty} \mid h^{0}\right)=w$, and $v\left(\sigma_{\infty} \mid h^{0}\right)=V_{\infty}$. Now we will construct such an optimal contract $\sigma_{\infty}$. By the definition of $\Phi(k, w)$, there exists a sequence of contracts $\left\{\sigma_{n}\right\}=\left\{a_{t}^{n}\left(h^{t-1}\right), c_{t}^{n}\left(h^{t}\right)\right\}$ and capital $\left\{k_{t+1}^{n}\left(h^{t-1}\right)\right\}$, where the constraints (2), (3), and (4), $\omega\left(\sigma_{n} \mid h^{0}\right)=w$ are satisfied for every $n$. Therefore

$$
V_{\infty}=\lim _{n \rightarrow \infty} \sum_{t=1}^{\infty} \beta^{t-1} \int\left[\theta_{t} f\left(k_{t}\right)-c_{t}^{n}\left(h^{t}\right)-k_{t+1}^{n}\left(h^{t-1}\right)+(1-\delta) k_{t}\right] g\left(\theta_{t} \mid a_{t}^{n}\left(h^{t-1}\right)\right) d h^{t}
$$

For $t=1$, notice that $\left\{a_{1}^{n}\left(h^{0}\right), c_{1}^{n}\left(h^{1}\right)\right\}$ and $\left\{k_{2}^{n}\left(h^{0}\right)\right\}$ are finite collections of bounded sequences. Therefore, there exist collections of subsequences $\left\{a_{1}^{n_{q}}\left(h^{0}\right), c_{1}^{n_{q}}\left(h^{1}\right)\right\}$ and $\left\{k_{2}^{n_{q}}\left(h^{0}\right)\right\}$ such that

$$
\lim _{n_{q} \rightarrow \infty} a_{1}^{n_{q}}\left(h^{0}\right)=a_{1}^{\infty}\left(h^{0}\right), \lim _{n_{q} \rightarrow \infty} c_{1}^{n_{q}}\left(h^{1}\right)=c_{1}^{\infty}\left(h^{1}\right), \text { and } \lim _{n_{q} \rightarrow \infty} k_{2}^{n_{q}}\left(h^{0}\right)=k_{2}^{\infty}\left(h^{0}\right) .
$$

We now consider $t=2$. Notice that $\left\{a_{2}^{n}\left(h^{1}\right), c_{2}^{n}\left(h^{2}\right)\right\}$ and $\left\{k_{3}^{n}\left(h^{1}\right)\right\}$ are finite collections of bounded sequences, and we can define $\left\{a_{2}^{\infty}\left(h^{1}\right), c_{2}^{\infty}\left(h^{2}\right)\right\}$ and $\left\{k_{3}^{\infty}\left(h^{1}\right\}\right.$ similarly as we did for $t=1$. If we iterate this procedure for $t=3,4, \ldots$, and let $\sigma_{\infty}=\left\{a_{t}^{\infty}\left(h^{t-1}\right), c_{t}^{\infty}\left(h^{t}\right)\right\}$ along with $k=\left\{k_{t+1}^{\infty}\left(h^{t-1}\right)\right\}$, then it is easy to verify that the constructed contract $\sigma_{\infty}$ is what we desired for.

\section{Proof of Proposition 2.}

Fix $\omega$, the lifetime discounted utility ensured by the optimal contract to the agent, and $k$, the optimal capital level of the firm. First, we show that $T\left(v^{*}\right)(k, \omega) \leq v^{*}(k, \omega)$. This inequality is true if there exists a feasible and incentive compatible contract $\sigma$ such that $\omega\left(\sigma \mid h^{0}\right)=\omega$ and $v\left(\sigma \mid h^{0}\right)=T\left(v^{*}\right)(k, \omega)$. The desired contract $\sigma$ can be constructed in the following way. Let $a(k, \omega), c(\theta, k, \omega), k^{\prime}(k, \omega)$, and $\omega^{\prime}(\theta, k, \omega)$ denote the solution of the maximization problem associated with the definition of $T\left(v^{*}\right)(k, \omega)$. Now, let $a_{1}\left(h^{0}\right)=a(k, \omega), c_{1}\left(h^{1}\right)=c\left(\theta_{1}, k, \omega\right)$, and $k_{2}\left(h^{0}\right)=k^{\prime}(k, \omega)$, $\forall h^{1}$ for $k=k_{1}$ given. For the realization of $\theta$ in $t=1$, denoted $\theta_{1}$ for the purpose of this proof, there exists a feasible and incentive compatible contract $\sigma_{\theta_{1}}$ that ensures a level of expected discounted utility $\omega^{\prime}\left(\theta_{1}, k, \omega\right)$ to the agent, and $v^{*}\left(k^{\prime}(k, \omega), \omega^{\prime}\left(\theta_{1}, k, \omega\right)\right)$ to the principal. Thus, we can say that $\sigma \mid h^{1}=\sigma_{\theta_{1}}, \forall h^{1}$. It is obvious that the constructed contract $\sigma$ is what is desired. 
We now need to show that $v^{*}(k, \omega) \leq T\left(v^{*}\right)(k, \omega)$. Let $\sigma^{*}$ be an optimal contract that ensures a level of expected discounted utility of $\omega$ to the agent, given $k$. In consequence, we can say that

$$
v^{*}(k, \omega)=v\left(\sigma^{*} \mid h^{0}\right)
$$

or

$v^{*}(k, \omega)=\int_{\Theta}\left\{\theta_{1} f\left(k_{1}\right)-c_{1}^{*}\left(\theta_{1}\right)-k_{2}^{*}\left(h^{0}\right)+(1-\delta) k_{1}+\beta v^{*}\left(k_{2}^{*}\left(h^{0}\right), \sigma^{*} \mid h^{1}\right)\right\} g\left(\theta \mid a_{1}^{*}\left(h^{0}\right)\right) d \theta$, or, finally,

$$
v^{*}(k, \omega) \leq T\left(v^{*}\right)(k, \omega),
$$

where the last inequality is obtained by letting $a(k, \omega)=a^{*}\left(h^{0}, k_{1}\right), c(\theta, k, \omega)=$ $c_{1}^{*}\left(\theta_{1}, k_{1}\right), \omega^{\prime}(\theta, k, \omega)=\omega^{\prime}\left(\sigma^{*} \mid h^{0}\right)$ along with $k^{\prime}(k, \omega)=k_{2}^{*}\left(h^{0}, k_{1}\right)$, for given $k=k_{1}$. This solution satisfies the constraints (5), (6), (7), and (8).

Notice that the operator $B$ is monotone, i.e. $\Sigma_{1} \subseteq \Sigma_{2}$ implies that $B\left(\Sigma_{1}\right) \subseteq B\left(\Sigma_{2}\right)$. Following Abreu, Pierce, and Stacchetti (1990), we say that $\Sigma$ is self-generating if $\Sigma \subseteq B(\Sigma)$.

Lemma 2 (a) $\Omega$ is self-generating. (b) If $\Sigma$ is self-generating, then $B(\Sigma) \subseteq \Omega$.

Proof. To prove (a), let $(k, \omega) \in \Omega$. There exists a contract $\sigma=\left\{a_{t}\left(h^{t-1}\right), c_{t}\left(h^{t}\right)\right\}$ and a sequence $\left\{k_{t+1}\left(h^{t-1}\right)\right\}$ which satisfy the constraints (2), (3), (4), and $\omega\left(\sigma \mid h^{0}\right)=\omega$. We now say that

$a(k, \omega)=a_{1}\left(h^{0}\right) ; \quad k^{\prime}(k, \omega)=k_{2}\left(h^{0}\right) ; c(\theta, k, \omega)=c_{1}(\{\theta\}), \forall \theta ; \omega^{\prime}(\theta, k, \omega)=\omega_{2}(\sigma \mid\{\theta\}), \forall \theta$.

It is obvious that $\left\{a(k, \omega), c(\theta, k, \omega), k^{\prime}(k, \omega), \omega^{\prime}(\theta, k, \omega)\right\}$, defined above, satisfies the constraints (5), (6),(7), and (8). Therefore, $(k, \omega) \in B(\Omega)$, which demonstrates that (a).

To prove (b), let $\Sigma$ be self-generating, and let $(k, \omega)_{h^{0}} \in B(\Sigma)$. We have to construct a contract $\sigma=\left\{a_{t}\left(h^{t-1}\right), c_{t}\left(h^{t}\right)\right\}$ and a sequence $k_{t+1}\left(h^{t-1}\right)=k_{h^{0}}$ that satisfy the constraints (2), (3), (4), and $\omega\left(\sigma \mid h^{0}\right)=\omega_{h^{0}}$. We construct such a contract recursively. First, there exist $\left\{a\left(k_{h^{0}}, \omega_{h^{0}}\right), c\left(\theta, k_{h^{0}}, \omega_{h^{0}}\right), k^{\prime}\left(k_{h^{0}}, \omega_{-} h^{0}\right), \omega^{\prime}\left(\theta, k_{h^{0}}, \omega_{h^{0}}\right)\right\}$ that satisfies (6), (8), and

$$
\begin{aligned}
& \int_{\Theta}\left\{u\left(c\left(\theta, k_{h^{0}}, \omega_{h^{0}}\right), m\left(a\left(k_{h^{0}}, \omega_{h^{0}}\right)\right) l(k)\right)+\beta \omega^{\prime}\left(\theta, k_{h^{0}}, \omega_{h^{0}}\right)\right\} g\left(\theta \mid a\left(k_{h^{0}}, w \omega_{h^{0}}\right)\right) d \theta=\omega_{h^{0}}, \\
& 0 \leq c\left(\theta, k_{h^{0}}, \omega_{h^{0}}\right) \leq \theta f(k)-k_{h^{0}}+(1-\delta) k .
\end{aligned}
$$


For $t=1$, let $a_{1}\left(h^{0}\right)=a\left(k_{h^{0}}, \omega_{h^{0}}\right)$ and $c 1\left(h^{1}\right)=c\left(\theta_{1}, k_{h^{0}}, \omega_{h^{0}}\right), \forall h^{1}$. Also, let $k_{h^{0}}^{\prime}=k_{h^{1}}=k^{\prime}\left(k_{h^{0}}, \omega_{h^{0}}\right)$ and $\omega_{h^{1}}=\omega^{\prime}\left(\theta, k_{h^{0}}, \omega_{h^{0}}\right), \forall h^{1}$. Notice that $\left(k_{h^{1}}, \omega_{h^{1}}\right) \in \Sigma \in$ $B(\Sigma)$ implies the existence of $\left\{a\left(k_{h^{1}}, \omega_{h^{1}}\right), c\left(\theta, k_{h^{1}}, \omega_{h^{1}}\right), k^{\prime}\left(k_{h^{1}}, \omega_{h^{1}}\right), \omega^{\prime}\left(\theta, k_{h^{1}}, \omega_{h^{1}}\right)\right\}$ that satisfies (6), (8), and

$$
\begin{aligned}
& \int_{\Theta}\left\{u\left(c\left(\theta, k_{h^{1}}, \omega_{h^{1}}\right), m\left(a\left(k_{h^{1}}, \omega_{h^{1}}\right)\right) l(k)\right)+\beta \omega^{\prime}\left(\theta, k_{h^{1}}, \omega_{h^{1}}\right)\right\} g\left(\theta \mid a\left(k_{h^{1}}, \omega_{h^{1}}\right)\right) d \theta=\omega_{h^{1}}, \\
& 0 \leq c\left(\theta, k_{h^{1}}, \omega_{h^{1}}\right) \leq \theta f(k)-k_{h^{1}}+(1-\delta) k .
\end{aligned}
$$

We can iterate for $t=2,3,4, \ldots$ to construct the complete profile $\sigma$. We can then observe that, by construction, for any arbitrary $t \geq 0$ and $h^{t}$,

$$
\omega\left(\sigma \mid h^{t}\right)-\omega_{h^{t}}=\int_{\Theta} \beta\left[\omega\left(\sigma \mid h^{t * 1}\right)-\omega_{h^{t * 1}}\right] g\left(\theta \mid a\left(k_{h^{t}}, \omega_{h^{t}}\right)\right) d \theta_{t * 1}
$$

Since $0<\beta<1$ and the utilities are bounded, the above equation implies that

$$
\omega\left(\sigma \mid h^{t}\right)=\omega_{h^{t}} \quad \forall t \geq 0 \text { and } \forall h^{t} .
$$

Hence, the contract that we have constructed is what is desired.

\section{Proof of Proposition 3.}

Part (a) is obvious. To show part (b), we will first show that the sequence $\left\{X_{n}\right\}$ is convergent. Obviously, $B\left(X_{0}\right) \subseteq X_{0}$. Next, we operate $B$ on both sides of this expression and obtain $X_{n+1}=B\left(X_{n}\right) \subseteq X_{n}, \forall n$, because $B$ is monotone increasing. Hence, $\left\{X_{n}\right\}$ is a bounded and monotone decreasing set sequence with $X_{\infty}=\lim _{n \rightarrow \infty} X_{n}=\bigcap_{n=0}^{\infty} X_{n}$. Now, we show that $\Omega \subseteq X_{\infty}$. Given that $\Omega \subseteq X_{0}$, the monotonicity property of $B$ ensures that $B(\Omega) \subseteq B\left(X_{0}\right)$. However, it must be true that $\Omega=B(\Omega)$, by part (a), and $B\left(X_{0}\right)=X_{1}$, by construction. Then, $\Omega \subseteq X_{1}$. By iteration we obtain $\Omega \subseteq X_{n}$, $\forall n \geq 0$, and consequently, $\Omega \subseteq X_{\infty}$. Now, we demonstrate that $X_{\infty} \subseteq \Omega$. Given the properties of the sequence $\left\{X_{n}\right\}$, we have that $B\left(X_{\infty}\right)=X_{\infty}$. Hence, $X_{\infty}$ is self-generating, and $X_{\infty}=B\left(X_{\infty}\right) \subseteq \Omega$.

Lemma 3 Let $k \geq\left(\theta_{l} / \delta\right)^{1 /(1-\alpha)}$. If $\bar{u}_{h}-\bar{u}_{l}<1$, then $\bar{\omega}(k)=\bar{u}_{l}$. If $\bar{u}_{h}-\bar{u}_{l}>1$, then $\bar{\omega}(k)=\bar{u}_{h}-1-\log \left(\bar{u}_{h}-\bar{u}_{l}\right)$. For $\chi<1, \underline{\omega}(k)=0$. For $\chi \geq 1, \underline{\omega}(k)=-\infty$.

Proof. The value $\bar{\omega}(k)$ is given by

$$
\begin{gathered}
\bar{\omega}(k)=\max _{u_{h}, u_{l}}\left(1-e^{-a^{*}}\right) u_{h}+e^{-a^{*}} u_{l}-a^{*} \\
\text { s.t. } a^{*}=\arg \max _{a}\left(1-e^{-a}\right) u_{h}+e^{-a} u_{l}-a, \\
\underline{u} \leq u_{i} \leq \bar{u}_{i} \quad i=h, l .
\end{gathered}
$$


Conditional on the optimal level of effort being zero, the solution calls for $u_{l}=\bar{u}_{l}$. When effort is strictly positive, then problem rewrites as

$$
\bar{\omega}(k)=\max _{u_{h}, u_{l}} u_{h}-1-\log \left(u_{h}-u_{l}\right)
$$

and the solution calls for $u_{i}=\bar{u}_{i}, i=h, l$. Since $u_{h}-u_{l}>1$ implies $u_{h}-1-\log \left(u_{h}-\right.$ $\left.u_{l}\right)>u_{l}$ it follows that $\bar{\omega}(k)=\bar{u}_{l}$ for $\bar{u}_{h}-\bar{u}_{l}<1$ and $\bar{\omega}(k)=\bar{u}_{h}-1-\log \left(\bar{u}_{h}-\bar{u}_{l}\right)$ otherwise.

Now turn to $\underline{\omega}(k)$. For $\chi>1$, any level of utility $\omega<\bar{u}_{l}$ can be awarded by setting $a^{*}=0$ and $c_{l}=c(\omega)$. Such allocation is feasible and incentive compatible. For $\chi<1$, any $\omega$ such that $0 \leq \omega \leq \bar{u}_{l}$ can be delivered in exactly the same way. Therefore, $\underline{\omega}(k) \leq 0$. By contradiction, assume that $\underline{\omega}(k)<0$. Obviously, this must happen for $a^{*}>0$. However, in this case promised utility is $u_{l}+e^{a^{*}}-1-a^{*}$, which is always non negative for $a^{*}>0$.

For any $k>0$, let $\omega_{b}(k)$ be equal to the infimum of the set of values $\omega$ such that the constraint $u_{i} \geq \underline{u}$ does not bind. It is easy to see that such set is always non-empty.

Proposition 5 When recommended effort is positive, $a^{*}=\log \left(u_{h}-u_{l}\right) \geq 0$. Furthermore:

(a) if $\chi \geq 1 / 2$, $a^{*}$ is strictly decreasing in $\omega$ and $u_{h}$ and $u_{l}$ are strictly increasing in $\omega$ for all $\omega$ such that the solution is interior;

(b) assume $u_{l} \leq \bar{u}_{l}$ binds; if $\bar{u}_{h}-\bar{u}_{l}<1$, then $a^{*}=0$, otherwise $a^{*}=\log \left(u_{h}^{*}-\bar{u}_{l}\right)$, where $u_{h}^{*}$ satisfies $u_{h}^{*}-1-\log \left(u_{h}^{*}-\bar{u}_{l}\right)-\omega$;

(c) let $\chi<1$; if $\left[\theta_{h}-\theta_{l}\right] k^{\alpha}>c(1)$, there exists $\omega_{r}>0$ such that $u_{l}=0$ and $u_{h}-1-\log \left(u_{h}\right)=\omega$ for all $\omega \in\left[0, \omega_{r}\right]$. If $\left[\theta_{h}-\theta_{l}\right] k^{\alpha} \leq c(1)$, then $a^{*}=0$ and $u_{l}=\omega$ for all $\omega$.

Proof. To prove (a), consider the necessary condition for an interior solution:

$$
\left[\theta_{h}-\theta_{l}\right] k^{\alpha}-\left[c\left(u_{h}\right)-c\left(u_{l}\right)\right]-(s-1)\left[c^{\prime}\left(u_{h}\right)-c^{\prime}\left(u_{l}\right)\right]=0 .
$$

This condition is also sufficient if

$$
-\left[c^{\prime}\left(u_{h}\right) \frac{1}{s}-c^{\prime}\left(u_{l}\right)\left(\frac{1}{s}-1\right)\right]-\left[c^{\prime}\left(u_{h}\right)-c^{\prime}\left(u_{l}\right)\right]-(s-1)\left[c^{\prime \prime}\left(u_{h}\right) \frac{1}{s}-c^{\prime \prime}\left(u_{l}\right)\left(\frac{1}{s}-1\right)\right]<0 .
$$

When $c^{\prime}(\cdot)$ is convex, sufficiency follows from the convexity of the cost function. This happens for $\chi \geq 1 / 2$. When $c^{\prime}(\cdot)$ is concave $(\chi<1 / 2)$, it follows from the fact that 
$c^{\prime \prime}(u)=\frac{\chi}{1-\chi} \frac{c^{\prime}(u)}{u}$. Applying the implicit function theorem to (24) reveals that $\chi \geq 1 / 2$ is sufficient, although not necessary condition for $\frac{d s}{d \omega}<0$. In turn, this directly implies $\frac{d u_{l}}{d \omega}>0$. To see that $\frac{d u_{h}}{d \omega}>0$ must hold, start from a pair $(k, \omega)$ and consider an infinitesimal increase in $\omega$. The left-hand side of (24) does not change. Since $\frac{d s}{d \omega}<0$ and $\frac{d u_{l}}{d \omega}>0$, for the right-hand side to remain unchanged it must be that $\frac{d u_{h}}{d \omega}>0$.

When $\bar{u}_{h}-\bar{u}_{l}<1$ and $\omega$ is such that $u_{l}=\bar{u}_{l}$, a positive effort recommendation is not incentive compatible. This is not the case when $\bar{u}_{h}-\bar{u}_{l}>1$. In such scenario, any increase in $\omega$ must be accommodated by raising $u_{h}$. This shows (b).

Now let $\chi<1$. For $\omega=0$, it is obvious that $a^{*}=0$ and $u_{h}=u_{l}=0$. In fact, if it were the case that $a^{*}>0,(24)$ would have to hold for $s>1$, which in turn would imply $u_{l}<0$. Now consider an infinitesimal increase in $\omega$. The constraint $s-\log (s) \leq 1+\omega$ relaxes. By continuity, however, it will still be the case that the left-hand side of (24) is still strictly positive over the admissible range of $s$. Since the left-hand side of (24) is decreasing in $\omega$, when $\left[\theta_{h}-\theta_{l}\right] k^{\alpha}>c(1)$ there will be a positive value of $\omega$ such that (24) holds true for $s$ such that $s-\log (s) \leq 1+\omega$. On the other hand, if $\left[\theta_{h}-\theta_{l}\right] k^{\alpha} \leq c(1)$, this will never be the case.

Proof of Proposition 4. Claims (a) and (b) are proven in the main body of the paper. Strict concavity follows from Theorem 4.8 in Stokey and Lucas (1989). To see why this is the case, rewrite the optimization program in (P2) as the operator $\mathcal{T}$, which maps the set of bounded and continuous functions defined over $[0, \bar{\omega}]$ into itself:

$$
\begin{aligned}
(\mathcal{T} v)(\omega) \equiv \max _{s \geq 1, \omega_{h}, \omega_{l}} & \left(1-\frac{1}{s}\right)\left[\theta_{h} k^{\alpha}-c\left(\omega+1+\log (s)-\beta \omega_{h}\right)+\beta v\left(\omega_{h}\right)\right]+ \\
& +\left(\frac{1}{s}\right)\left[\theta_{l} k^{\alpha}-c\left(\omega+1+\log (s)-s-\beta \omega_{l}\right)+\beta v\left(\omega_{l}\right)\right]-\delta k, \\
\text { s.t. } & \omega+1+\log (s)-\beta \omega_{h} \geq 0, \\
& \omega+1+\log (s)-s-\beta \omega_{l} \geq 0, \\
& 0 \leq \omega_{i} \leq \bar{\omega} \forall i=h, l .
\end{aligned}
$$

First, notice that since $a^{*} \in[0, \bar{a}]$ and $a=\max [0, \log (s)], s$ is bounded. This implies that the choices of $u_{h}$ and $u_{l}$ are also bounded. Furthermore, the constraint set is convex, in the following sense. Let $\Gamma(\omega)$ denote the set of feasible triplets $\kappa=$ $\left(s, \omega_{h}, \omega_{l}\right)$ when the state is $\omega$. For all pairs $\omega_{a}, \omega_{b} \in[0, \bar{\omega}]$ and all $\left\{\kappa_{a}, \kappa_{b}\right\}$ such that $\kappa_{a} \in \Gamma\left(\omega_{a}\right)$ and $\kappa_{b} \in \Gamma\left(\omega_{b}\right), \eta \kappa_{a}+(1-\eta) \kappa_{b} \in \Gamma\left[\eta \omega_{a}+(1-\eta) \omega_{b}\right]$ for all $\eta \in[0,1]$.

Finally, strict convexity of the function $c$ guarantees that for all concave, bounded 
and continuous functions $v$ defined over $[0, \bar{\omega}],(\mathcal{T})(v)\left(\eta \omega_{a}+(1-\eta) \omega_{b}\right)>\eta(\mathcal{T})(v)\left(\omega_{a}\right)+$ $(1-\eta)(\mathcal{T})(v)\left(\omega_{b}\right)$, for all $\omega_{a}, \omega_{b} \in[0, \bar{\omega}]$ and all $\eta \in[0,1]$.

\section{B Algorithm}

In this section we provide a brief description of the algorithm that was used to compute a numerical approximation to the value function $v(k, \omega)$. Given that the set $\Omega$ is not square, it is not efficient to approximate the value function by means of bi-dimensional splines. For this reason, we will restrict the choice of capital to a finite number of levels and approximate the value function on the $\omega$ dimension by means of cubic splines.

We start by defining a fine grid for the capital stock. Denote it as $\mathcal{K} \equiv\left\{k_{j}\right\}_{j=1}^{n_{k}}$ and let the related set of indexes be $\mathcal{J} \equiv\{j\}_{j=1}^{n_{k}}$. The upper bound of $\mathcal{K}$ must be chosen in such a way that the corresponding net investment will be negative for all $\omega$. For this to be the case, it is sufficient to set it equal to the efficient capital stock when

$\theta=\theta_{h}$ with probability 1 in all periods. That is, we let $k_{n_{k}}=\left(\frac{\alpha \theta_{h}}{\delta}\right)^{\frac{1}{1-\alpha}}$. The next task consists in approximating the equilibrium value set of the transformed problem.

\section{B.1 Approximation of the Set $\Omega$}

From the analysis conducted in Section 2, it follows that for every $j \in \mathcal{J}$, the set of feasible and incentive compatible values will be given by an interval $\left[\underline{\omega}, \bar{\omega}_{j}\right] \in \Re_{+}$. This means that our task reduces to approximate the mapping $\Omega: \mathcal{K} \rightarrow \Re_{+}$which is given by the sequence $\left\{\bar{\omega}_{j}\right\}_{j \in \mathcal{J}}$. The mapping $\Omega$ can be shown to be increasing and strictly concave.

Following Abreu, Pierce, and Stacchetti (1990), we start by defining an initial guess $\Omega_{0}=\left\{\bar{\omega}_{0 j}\right\}_{j \in \mathcal{J}}$. We impose that $\Omega_{0}$ is weakly increasing, weakly concave, and such that $\bar{\omega}_{0 j} \geq \bar{\omega}_{j}$ for all $j$. These requirements are satisfied by letting $\bar{\omega}_{0 j}=$ $\frac{u\left(\theta_{h} k_{n_{k}}^{\alpha}-\delta k_{n_{k}}\right)}{1-\beta}$. Then, for every $j, q \in \mathcal{J}$ such that $\theta_{l} k_{j}^{\alpha}+k_{j}(1-\delta)-k_{q} \geq 0$, we compute

$$
\begin{aligned}
b_{j q} \equiv \max _{a,\left\{u_{i}, \omega_{i}\right\}_{i=h, l}} & \left(1-e^{-a}\right)\left[u_{h}+\beta \omega_{h}\right]+e^{-a}\left[u_{l}+\beta \omega_{l}\right]-a \\
\text { s.t. } 0 & \leq u_{i} \leq u\left(\theta_{i} k_{j}^{\alpha}+k_{j}(1-\delta)-k_{q}\right), \\
\underline{\omega} & \leq \omega_{i} \leq \bar{\omega}_{n q}
\end{aligned}
$$

and

$$
\bar{\omega}_{n+1, j} \equiv \max _{j}\left\{b_{j q}\right\}
$$


The operator defined by (25)-(26) generates a sequence $\left\{\Omega_{n}\right\}$ that converges to $\Omega$. Our approximation will be $\Omega_{m}$ such that $\left\|\Omega_{m}-\Omega_{m-1}\right\|_{\infty}<10.0^{-8}$.

Notice that, conditional on effort being zero, the above optimization problem simplifies to

$$
\begin{array}{ll}
\max _{u_{l}, \omega_{l}} & u_{l}+\beta \omega_{l} \\
\text { s.t. } & 0 \leq u_{l} \leq u\left(\theta_{l} k^{\alpha}+k(1-\delta)-k_{j}\right), \\
& \underline{\omega} \leq \omega_{l} \leq \bar{\omega}_{n j} .
\end{array}
$$

Obviously the solution calls for $u_{l}=u\left(\theta_{l} k_{j}^{\alpha}+k_{j}(1-\delta)-k_{q}\right)$ and $\omega_{l}=\bar{\omega}_{n q}$. Alternatively, when effort is strictly positive, $a=\log (s)$, where $s \equiv\left(u_{h}+\beta \omega_{h}-u_{l}-\beta \omega_{l}\right)$. The optimization problem then becomes

$$
\begin{aligned}
& \max _{s, u_{h}, \omega_{h}} u_{h}+\beta \omega_{h}-1-\log (s) \\
& \text { s.t. } 0 \leq u_{i} \leq u\left(\theta_{i} k_{j}^{\alpha}+k_{j}(1-\delta)-k_{q}\right), \\
& \quad \underline{\omega} \leq \omega_{i} \leq \bar{\omega}_{n q} .
\end{aligned}
$$

In this case the solution calls for $u_{i}=u\left(\theta_{i} k_{j}^{\alpha}+k_{j}(1-\delta)-k_{q}\right)$ and $\omega_{i}=\bar{\omega}_{n q}$.

\section{B.2 Approximation of the Value Function}

For every $j \in \mathcal{J}$, we define a coarse grid $\mathcal{Z}_{j}=\left\{\omega_{j z}\right\}_{z=1}^{n_{\omega}}$ over the interval $\left[\underline{\omega}, \bar{\omega}_{j}\right]$. We also define an initial guess for the value function: $v_{0 j}: \mathcal{Z}_{j} \rightarrow \Re_{+}$. For all other $\omega \in\left[\underline{\omega}, \bar{\omega}_{j}\right]$, the guess is approximated by a cubic spline which we denote as $v_{0 j}(\omega)$. We impose that $v_{0 j}(\omega)$ is decreasing and concave in $\omega$ for all $j \in \mathcal{J}$ and that the function is increasing and concave in capital. Then, for all $z$ and every $j, q \in \mathcal{J}$ such that $\theta_{l} k_{j}^{\alpha}+k_{j}(1-\delta)-k_{q} \geq 0$, we compute

$$
\begin{aligned}
d_{j z q} \equiv \max _{a^{*},\left\{u_{i}, \omega_{i},\right\}_{i=h, l}} & \left(1-e^{-a^{*}}\right)\left[\theta_{h} k^{\alpha}-c\left(u_{h}\right)+\beta v_{n q}\left(\omega_{h}\right)\right]+e^{-a^{*}}\left[\theta_{l} k^{\alpha}-c\left(u_{l}\right)+\beta v_{n q}\left(\omega_{l}\right)\right] \\
& +k_{j}(1-\delta)-k_{q} \\
\text { s.t. } & \left(1-e^{-a^{*}}\right)\left[u_{h}+\beta \omega_{h}\right]+e^{-a^{*}}\left[u_{l}+\beta \omega_{l}\right]-a^{*}=\omega_{j z} \\
& a^{*}=\arg \max \left(1-e^{-a}\right)\left[u_{h}+\beta \omega_{h}\right]+e^{-a}\left[u_{l}+\beta \omega_{l}\right]-a \\
& 0 \leq u_{i} \leq u\left(\theta_{i} k_{j}^{\alpha}+k_{j}(1-\delta)-k_{q}\right) \forall i=h, l \\
& a^{*} \geq 0 \\
& \underline{\omega} \leq \omega_{i} \leq \bar{\omega}_{q} \forall i=h, l
\end{aligned}
$$


and

$$
v_{n+1, j z} \equiv \max _{q}\left\{d_{j z q}\right\} .
$$

The operator defined by (27)-(28) generates a sequence $\mathcal{V}_{n} \equiv\left\{v_{n j}\right\}_{j \in \mathcal{J}}$. Our approximation of the value function on the grid will be $\mathcal{V}_{m}$ such that $\left\|\mathcal{V}_{m}-\mathcal{V}_{m-1}\right\|_{\infty}<$ $10.0^{-8}$. Notice that when recommended effort is positive, the above optimization problem simplifies to

$$
\begin{aligned}
\max _{s,\left\{u_{i}\right\}_{i=h, l}}\left(1-\frac{1}{s}\right)\left[\theta_{h} k^{\alpha}-c\left(u_{h}\right)+\beta v_{n q}\left(\omega_{h}\right)\right]+\frac{1}{s}\left[\theta_{l} k^{\alpha}-c\left(u_{l}\right)+\beta v_{n q}\left(\omega_{l}\right)\right] \\
+k_{j}(1-\delta)-k_{q}, \\
\text { s.t. } \omega_{h}=\left[\omega_{j z}+1+\log (s)-u_{h}\right] / \beta, \\
\omega_{l}=\left[\omega_{j z}+1+\log (s)-s-u_{l}\right] / \beta, \\
0 \leq u_{i} \leq u\left(\theta_{i} k_{j}^{\alpha}+k_{j}(1-\delta)-k_{q}\right) \forall i=h, l, \\
\underline{\omega} \leq \omega_{i} \leq \bar{\omega}_{q} \forall i=h, l .
\end{aligned}
$$

\section{References}

Abreu, D., D. Pierce, and E. Stacchetti (1990): "Toward a Theory of Discounted Repeated Games with Imperfect Monitoring," Econometrica, 58, 10401063.

Aggarwal, R., and M. Gort (1996): "The Evolution of Markets and Entry, Exit and Survival of Firms," Review of Economics and Statistics, 78(3), 489-498.

- (2002): "Firm and Product Life Cycles and Firm Survival," American Economic Review, 92(2), 184-190.

Albuquerque, R., and H. Hopenhayn (2004): "Optimal Lending Contracts and Firm Dynamics," Review of Economic Studies, 71, 285-315.

Atkeson, A., And R. Lucas (1995): "Efficiency and Equality in a Simple Model of Efficient Unemployment Insurance," Journal of Economic Theory, 66, 64-98.

Bahk, B., And M. Gort (1993): "Decomposing Learning by Doing in New Plants," Journal of Political Economy, 101(4), 561-583.

Bohacek, R. (2005): "Capital Accumulation in Private Information Economies," Topics in Macroeconomics, 5(1), Article 24. 
Brusco, S., And E. Ropero (2007): "Financing Constraints and Firm Dynamics with Durable Capital," Working paper, SUNY at Stony Brook.

Caves, R. E. (1998): "Industrial Organization and New Findings on the Turnover and Mobility of Firms," Journal of Economic Literature, 36(4), 1947-1982.

Choi, S., R. Fisman, D. Gale, and S. Kariv (2007): "Consistency and Heterogeneity of Individual Behavior under Uncertainty," American Economic Review, 97(5), 1921-1938.

Clementi, G. L., T. Cooley, and C. Wang (2006): "Stock Grants as a Commitment Device," Journal of Economic Dynamics and Control, 30(11), 2191-2216.

Clementi, G. L., and H. Hopenhayn (2006): "A Theory Financing Constraints and Firm Dynamics," Quarterly Journal of Economics, 121(1), 229-265.

Cooley, T., R. Marimon, and V. Quadrini (2004): "Aggregate Consequences of Limited Contract Enforceability," Journal of Political Economy, 112(4), 817-847.

Cooley, T., And V. QuAdrini (2001): "Financial Markets and Firm Dynamics," American Economic Review, 91(4), 1286-1310.

Demarzo, P., M. Fishman, Z. He, and N. Wang (2008): "Dynamic Agency and the q Theory of Investment," Working paper, Graduate School of Business, Stanford University.

Espino, E. (2005): "On Ramsey's Conjecture: Efficient Allocations in the Neoclassical Growth Model With Private Information," Journal of Economic Theory, 121, $192-213$.

Fernandes, A., And C. Phelan (2000): "A Recursive Formulation for Repeated Agency with History Dependence," Journal of Economic Theory, 91, 223-47.

Green, E. (1987): "Lending and the Smoothing of Uninsurable Income," in Contractual Arrangements for Intertemporal Trade. University of Minnesota Press, Minneapolis, MN.

Holmstrom, B. (1979): "Moral Hazard and Observability," Bell Journal of Economics, 10, 74-91.

Hopenhayn, H. (1992): "Entry, Exit, and Firm Dynamics in Long Run Equilibrium," Econometrica, 60(5), 1127-1150. 
Jovanovic, B. (1982): "Selection and the Evolution of Industry," Econometrica, 50(3), 649-670.

Khan, A., And B. Ravikumar (2001): "Growth and Risk-Sharing with Private Information," Journal of Monetary Economics, 47, 499-521.

Loderer, C., K. Neusser, and U. Waelchli (2009): "Firm Age and Survival," working paper, University of Bern.

Marcet, A., and R. Marimon (1992): "Communication, Commitment, and Growth," Journal of Economic Theory, 58, 219-249.

Newman, A. (2007): "Risk-Bearing and Entrepreneurship," Journal of Economic Theory, 137(1), 11-26.

QuAdrini, V. (2003): "Investment and Liquidation in Renegotiation-Proof Contracts with Moral Hazard," Journal of Monetary Economics, 51, 713-751.

RAmpini, A. (2004): "Entrepreneurial Activity, Risk, and the Business Cycle," Journal of Monetary Economics, 51, 555-573.

Rogerson, W. (1985a): "The First-Order Approach to Principal-Agent Problems," Econometrica, 53, 1357-1968.

(1985b): "Repeated Moral Hazard," Econometrica, 53, 69-76.

Spear, S., and S. Srivastava (1987): "On Repeated Moral Hazard with Discounting," Review of Economic Studies, 54, 599-617.

Spear, S., And C. Wang (2005): "When to Fire a CEO: Optimal Termination in Dynamic Contracts," Journal of Economic Theory, 120, 239-256.

Stokey, N. L., And R. J. Lucas (1989): Recursive Methods in Economic Dynamics. Harvard University Press, Cambridge, MA.

WAng, C. (1997): "Incentives, CEO Compensation, and Shareholder Wealth in a Dynamic Agency Model," Journal of Economic Theory, 76, 72-105.

(2006): "Equilibrium Layoff as Termination of a Dynamic Contract," Working Paper \# 06042, Iowa State. 